University of New Mexico

UNM Digital Repository

Mathematics and Statistics Faculty and Staff

Publications

Academic Department Resources

8-2020

\title{
A Neutrosophic Clinical Decision-Making System for Cardiovascular Diseases Risk Analysis
}

\author{
Florentin Smarandache \\ University of New Mexico, smarand@unm.edu \\ Shaista Habib \\ shaista.habib@umt.edu.pk \\ Wardat-us- Salam \\ wardahtussalam@gmail.com \\ M. Arif Butt \\ arif@pucit.edu.pk \\ Muhammad Akram \\ m.akram@pucit.edu.pk
}

Follow this and additional works at: https://digitalrepository.unm.edu/math_fsp

Part of the Analytical, Diagnostic and Therapeutic Techniques and Equipment Commons, Bioinformatics Commons, Health Information Technology Commons, Mathematics Commons, Medical

Sciences Commons, Other Life Sciences Commons, and the Other Medicine and Health Sciences

Commons

\section{Recommended Citation}

Smarandache, Florentin; Shaista Habib; Wardat-us- Salam; M. Arif Butt; and Muhammad Akram. "A Neutrosophic Clinical Decision-Making System for Cardiovascular Diseases Risk Analysis." JIFS (2020): 1-31. https://digitalrepository.unm.edu/math_fsp/459

This Article is brought to you for free and open access by the Academic Department Resources at UNM Digital Repository. It has been accepted for inclusion in Mathematics and Statistics Faculty and Staff Publications by an authorized administrator of UNM Digital Repository. For more information, please contact amywinter@unm.edu, Isloane@salud.unm.edu,sarahrk@unm.edu. 


\title{
A Neutrosophic Clinical Decision-Making System for Cardiovascular Diseases Risk Analysis
}

\author{
Shaista Habib ${ }^{a}$, Wardat-us-Salam ${ }^{b}$, M. Arif Butt ${ }^{c}$, M. Akram ${ }^{d}$ and F. Smarandache \\ a. School of Systems and Technology, University of Management and Technology, Lahore, Pakistan \\ E-mail: shaista.habib@umt.edu.pk \\ b. Department of Mathematics, Division of Science \& Technology, University of Education, Lahore, Pakistan. \\ E-mail: wardahtussalam@gmail.com \\ c. Punjab University College of Information Technology, University of the Punjab, Lahore-54000, Pakistan \\ E-mail: arif@pucit.edu.pk \\ d. Department of Mathematics, University of the Punjab, New Campus, Lahore-54590, Pakistan \\ E-mail: m.akram@pucit.edu.pk \\ e. Mathematics and Science Department, University of New Mexico, Gallup, NM 87301, USA. \\ E-mail: fsmarandache@gmail.com
}

August 7, 2020

\begin{abstract}
Cardiovascular diseases are the leading cause of death worldwide. Early diagnosis of heart disease can reduce this large number of deaths so that treatment can be carried out. Many decision-making systems have been developed, but they are too complex for medical professionals. To target these objectives, we develop an explainable neutrosophic clinical decision-making system for the timely diagnose of cardiovascular disease risk. We make our system transparent and easy to understand with the help of explainable artificial intelligence techniques so that medical professionals can easily adopt this system. Our system is taking thirtyfive symptoms as input parameters, which are, gender, age, genetic disposition, smoking, blood pressure, cholesterol, diabetes, body mass index, depression, unhealthy diet, metabolic disorder, physical inactivity, pre-eclampsia, rheumatoid arthritis, coffee consumption, pregnancy, rubella, drugs, tobacco, alcohol, heart defect, previous surgery/injury, thyroid, sleep apnea, atrial fibrillation, heart history, infection, homocysteine level, pericardial cysts, marfan syndrome, syphilis, inflammation, clots, cancer, and electrolyte imbalance and finds out the risk of coronary artery disease, cardiomyopathy, congenital heart disease, heart attack, heart arrhythmia, peripheral artery disease, aortic disease, pericardial disease, deep vein thrombosis, heart valve disease, and heart failure. There are five main modules of the system, which are neutrosophication, knowledge base, inference engine, de-neutrosophication, and explainability. To demonstrate the complete working of our system, we design an algorithm and calculates its time complexity. We also present a new de-neutrosophication formula, and give comparison of our the results with existing methods.
\end{abstract}

Keywords: Single-valued neutrosophic number, explainable artificial intelligence, cardiovascular diseases, decision making, de-neutrosophication, algorithm.

\section{Introduction}

Artificial intelligence (AI) is an umbrella term for algorithms aiming at delivering task of solving capabilities comparable to humans. Its dominant sub-field is decision-making to develop a system that can make an intelligent decision, on behalf of humans to improve the quality of life [1]-[3]. Even in the health care industry, 
automatic approaches recently demonstrated impressive findings. Unfortunately, such approaches have several disadvantages because of a lot of mathematical modeling, they are often considered black-box approaches, which do not foster trust and acceptance of AI generally. AI models or computational theories are generally considered not explainable [4]. In health care, there is a growing need for AI approaches, which are not only performing well but are trustworthy, transparent, and explainable for human experts in the health care industry [5]-[7].

According to the world health organization (WHO), cardiovascular diseases (CVDs) are the leading cause of death worldwide. About 17.9 million people die of heart diseases. With the help of different decision-making methods, it is possible to diagnose the disease at an early stage or at least guide a medical expert about the impending health condition, so that precautions can be taken and overall deaths can be reduced globally. Such a decision-making system will facilitate and simplifies the work of medical professionals. Many such systems have already been introduced in the literature, but because of the lack of transparency and the complexity of decision-making methods, it is difficult for medical professionals to adopt or use them with confidence. To achieve these goals, we introduced a new decision-making system which makes every step of the decision-making process transparent using understandable AI techniques, so that the medical expert understands that how the decision-making process works.

The information related to the diseases contains a-lot of imperfections, vagueness, and imprecision which may lead to an incorrect diagnosis. In 1965, Zadeh proposed a novel concept named fuzzy sets to deal with vagueness and imprecisions [8]. After that, many extensions of fuzzy sets derived and neutrosophic sets (NSs) are also one of them [9]. Smarandache proposed as a new branch of philosophy called neutrosophy in 1999 [10]. Neutrosophy is the base of the neutrosophic set (NS) and neutrosophic logic (NL). NS simultaneously focuses on true membership, falsity membership, and indeterminacy membership, which are more practical and appropriate than fuzzy systems and intuitionistic fuzzy systems in trade, which are unsatisfactory, incomplete, and is inconsistent in sequence. The single-valued neutrosophic set (SVNS) is an extension of the NS [11]-[13]. Ye introduced [14] simplified neutrosophic sets, and Peng et al. [21,22] explained precisely their new operation and aggregate operators. The neutrosophic logic (NL) has no restriction on truth membership, indeterminacy membership, and falsity membership, while intuitionistic fuzzy logic has a summation of its components (or their upper limit) $=1$. In this way, neutrosophic logic can address incomplete information $(\operatorname{sum}<1)$, and para-consistent information $(\operatorname{sum}>1)$. This property of neutrosophic sets makes it more suitable for the interpretation of medical knowledge.

There are many decision-making methods proposed in the literature, some of them are discussed here. The function of sign trigonometry (ST) is a method of decision making, which plays an important role in the aggregation of data. The biggest advantages of this function are its periodicity and the fact that it is symmetric about the origin, and therefore it meets the decision-making priorities over the multi-time phase parameters [15]. Immediate probabilities aggregation operators for single-valued and interval neutrosophic sets is another method of decision making which describes the decision-maker's behavior objectively (in terms of probability) and subjectively (in terms of weight), with the concept of probabilistic information playing a dominant role in the investigation. The advantage of these proposed operators is that they simultaneously combine objective and subjective behavior in the decision-making process [16].

The technique for order preference by similarity to an ideal solution (TOPSIS) is a well-known approach that works on the principle of making the best choice according to the minimum distance from the target. For this, PIS ("Positive Ideal Set") and NIS ("Negative Ideal Set") two theories are considered and depends on the method of TOPSIS. In the TOPSIS method, both inclinations, such as similarity or dissimilarity, are looked all together to achieve the goal. Based on these features, numerous researchers have solved the problem of TOPSIS to solve the problem of multiple criteria decision making under the SVNS environment [17]. Frank tnorm operations are more compatible and common than other norms, giving the decision-maker more flexibility while adjusting its parametric value. Meanwhile, the correlation between any kind of argument is a significant advantage of the heroin main (HM) operator. Encouraged by these basic features, it is interesting to combine Choquet integral HM operator to the SVNS based on the Frank norm operations [18]. During the aggregation 
process, the most important process is to define the operational rules. But from the current literature, it is observed that most of the existing aggregation operators are based on the assumption that there is a crisp number within the weight [0.1]. However, Ye et al. introduced explicit operational rules as an appendix to the SVNS operational rules, where the bases are the real numbers and the exponents are the SVNS [19].

In 2019, Zhu et al. proposed a family of pythagorean fuzzy aggregation operators based on the interactive operational laws of pythagorean fuzzy numbers (PFNs). Such as pythagorean fuzzy Interaction power PBM (PFIPPBM), pythagorean fuzzy Interaction power partitioned geometric bonferroni mean (PFIPPGBM), and weighted forms of PFIPPBM and PFIPPGBM. Proposed operators can not only handle situations where attributes are divided into several sections and in which the attributes are interrelated but also reduced the negative impact of irrational reviews on the outcome of the decision. He performed numerical examples as well as a comparative analysis to show the validity and superiority of the proposed approach [20].

In 2016, Ye et al. [23] proposed a novel single-valued neutrosophic similarity measuring method to resolve multi-period medical diagnosis problems. This technique used the tangent function and weighted aggregation of multi-period data. They compared their formula generated results with other similarity measures with the help of pattern recognition examples. After that, a multi-period example is demonstrated to find out the applicability of a multi-period medical diagnostic method with the help of comprehensive information on a multi-period.

In 2019, Cui et al. [24] presented a dynamic neutrosophic cubic set (DNCS) as an extension of neutrosophic sets. They expressed the patient's symptom data using varying periods. Afterward, they used the proposed logarithmic similarity measure (LSM) of the DNCSs to diagnose the patient's disease. Subsequently, a clinical diagnosis method is developed that used a logarithmic similarity measure of DNCS, where information on the symptoms of the disease is gathered after fixed time intervals. The time intervals are identified by DNCS. The applicability of this technique is checked with the help of different examples.

In 2019, Basset et al. [25] offered a new way to evaluate the process of selecting smart medical devices (SMDs) in group decision-making (GDM) in an ambiguous decision environment. The proposed method combined neutrosophic bipolar numbers with order priority techniques. In this study, diabetes patients had taken to choose diabetes diagnostics smart medical devices. Their main objective was to present the complexities of the problem, increase interest among experts in the health care industry, and evaluate smart medical devices under different diagnostic criteria. The results of the neutrosophic are analyzed with the help of the TOPSIS model, which showed that the obtained results and classification capability are sufficiently stable. The results generated from the proposed techniques are also compared with other models.

In 2019, Gulerial et al. [26] demonstrated various decision-making models that are useful for handling impreciseness and uncertainty among the qualitative and quantitative factors of the decision-making process. In the proposed work, a new parametric divergence measure for neutrosophic sets has suggested along with its various characteristics. Based on this method parametric divergence measured and outlined some methodologies along with its implementing procedural steps for classification problems and multi-criteria decision-making problem. Also, numerical examples of the application problems have provided for the illustration of the proposed methodologies. They compared their results with existing approaches as well.

The motivations of this article are described below:

1. CVD is the leading cause of death worldwide. Deaths from CVD can be reduced if a decision can be made by experts earlier.

2. There is a lot of uncertainty in health-related data. Misdiagnosis can be made if we do not handle such data effectively.

3. Current decision-making systems are extremely complex, and lack transparency, making it difficult for medical professionals to understand them, so they are reluctant to adopt them with confidence. 
Our contribution to this article is outlined below:

1. We develop a new decision-making system to determine the risks of heart disease. This system early detects heart disease to reduce the overall mortality rate. The decision-making system is taking thirty-five parameters as input and determining the risks of eleven heart diseases.

2. SVNS is used for decision making. SVNS is very close to human thinking because it focuses on the degree of truth, the degree of indeterminacy, and the degree of falsity at the same time, and there is no restriction on its sum, unlike intuitionistic fuzzy sets.

3. We integrate the concept of explainable AI to make our system adaptable for medical professionals. The quality of explanation is measure using causability. Explainable AI and causability AI systems build the confidence of medical professionals.

4. We design the algorithms to show the complete working of the system, as well as calculate its time complexity.

5. We also propose a new de-neutrosophication formula as an alternative approach to existing methods and provide a comparative analysis with existing methods.

The remainder of this paper is organized in the following way: Section 2 briefly reviews important concepts of neutrosophic sets, explainable AI, and causability measures. Section 3 discusses the explainable neutrosophic clinical decision-making systems for cardiovascular diseases. Section 4 presents a case study to show the effectiveness of the system. Section 5 compares the results of the proposed de-neutrosophication formula with the existing de-neutrosophication formula. Section 6 concludes this paper and discusses possible future research directions.

\section{Preliminaries}

This section reviews some of the preliminary notions that need to be understood to fully benefit from this article.

2.1. Explainability [5]: Explainability highlights decision-relevant parts of the algorithms and the active parts of the algorithmic model, that either contribute to the model accuracy on the training set, or a specific prediction for one particular observation. It does not refer to an explicit human model. Two major explainable models can be used in medical domains. The first one is the post-hoc explainability. Posthoc systems aim to provide local explanations for a specific decision and make it reproducible on demand (instead of explaining the whole system's behavior); typical examples are LIME and BETA. Ante-hoc systems are interpretable by design towards glass-box approaches; typical examples include linear regression, decision trees, and fuzzy inference systems.

2.2. Causability [5]: Causability defines the extent to which an explanation of a statement of a human expert achieves a specified level of causal understanding with effectiveness, efficiency, and satisfaction in a specified context of use. In simple words, causability measures the quality of an explanation.

2.3. Single-valued neutrosophic sets [11]: Let $U$ be a space of points (objects). A single-valued neutrosophic set $S$ on a non-empty set $U$ is characterized by a truth membership function $T_{S}: U \rightarrow[0,1]$, indeterminacy membership function $I_{S}: U \rightarrow[0,1]$ and a falsity membership function $F_{S}: U \rightarrow[0,1]$. Thus, $S=\left\{<p, T_{S}(p), I_{S}(p), F_{S}(p)>\mid p \in U\right\}$. There is no restriction on the sum of $T_{S}(p), I_{S}(p)$ and $F_{S}(p)$ for all $p \in U$.

2.4. Neutrosophic logic [27]: Neutrosophic logic (NL) was presented by Smarandache as an extension of the fuzzy logic, intuitionistic logic, and para-consistent logic. This logic consists of three basic components, that is, membership of truth, membership of indeterminacy, and membership of falsity. 
2.5. Single-valued neutrosophic number [9]: Single-valued neutrosophic number $(A)$ is defined as $A=$ $\left(\left[\left(a_{1}, b_{1}, c_{1}, d_{1}\right) ; \rho\right],\left[\left(a_{2}, b_{2}, c_{2}, d_{2}\right) ; \sigma\right],\left[\left(a_{3}, b_{3}, c_{3}, d_{3}\right) ; \omega\right]\right)$ where $\rho, \sigma, \omega \in[0,1]$, the truth membership function $\left(\mu_{A}\right): \mathbb{R} \rightarrow[0, \rho]$, indeterminacy membership function $\left(\nu_{A}\right): \mathbb{R} \rightarrow[\sigma, 1]$, and falsity membership function $\left(\lambda_{A}\right): \mathbb{R} \rightarrow[\omega, 1]$ are written as follows:

$$
\begin{gathered}
\mu_{A}(p)= \begin{cases}\mu_{A l}(p), & \text { if } a_{1} \leq p \leq b_{1}, \\
\rho, & \text { if } b_{1} \leq p \leq c_{1}, \\
\mu_{A u}(p), & \text { if } c_{1} \leq p \leq d_{1}, \\
0, & \text { otherwise. }\end{cases} \\
\nu_{A}(p)= \begin{cases}\nu_{A l}(p), & \text { if } a_{2} \leq p \leq b_{2}, \\
\sigma, & \text { if } b_{2} \leq p \leq c_{2}, \\
\nu_{A u}(p), & \text { if } c_{2} \leq p \leq d_{2}, \\
1, & \text { otherwise. }\end{cases} \\
\lambda_{A}(p)= \begin{cases}\lambda_{A l}(p), & \text { if } a_{3} \leq p \leq b_{3}, \\
\omega, & \text { if } b_{3} \leq p \leq c_{3}, \\
\lambda_{A u}(p), & \text { if } c_{3} \leq p \leq d_{3}, \\
1, & \text { otherwise. }\end{cases}
\end{gathered}
$$

2.6. The intersection of two single-valued neutrosophic sets [11]: Let $\mathrm{P}$ be a space of points and $S_{1}$ and $S_{2}$ are the two single-valued neutrosophic sets and $S_{3}$ holds the result of intersection. The intersection of these two sets can be expressed as $S_{3}=S_{1} \cap S_{2}$, and the truth-membership function, indeterminacy-membership function, and falsity-membership function are as follows:

$$
\begin{gathered}
T_{S_{3}}(p)=\min \left(T_{S_{1}}(p), T_{S_{2}}(p)\right), \\
I_{S_{3}}(p)=\max \left(I_{S_{1}}(p), I_{S_{2}}(p)\right), \\
F_{S_{3}}(p)=\max \left(F_{S_{1}}(p), F_{S_{2}}(p)\right),
\end{gathered}
$$

for all $\mathrm{p}$ in $\mathrm{P}$.

2.7. Union of two single-valued neutrosophic sets [11]: If $S_{1}$ and $S_{2}$ are the two single-valued neutrosophic sets and $S_{3}$ holds the result of the union. Their union can be expressed as $S_{3}=S_{1} \cup S_{2}$, whose truth-membership, indeterminacy-membership and falsity-membership functions are as follows:

$$
T_{S_{3}}(p)=\max \left(T_{S_{1}}(p), T_{S_{2}}(p)\right), I_{S_{3}}(p)=\min \left(I_{S_{1}}(p), I_{S_{2}}(p)\right), F_{S_{3}}(p)=\min \left(F_{S_{1}}(p), F_{S_{2}}(p)\right),
$$

for all $\mathrm{p}$ in $\mathrm{P}$.

\subsection{Major factors of cardiovascular diseases [28]-[31]:}

Genetic disposition (GD): GD is the increases in the likelihood of developing a particular disease depending upon a person's genes.

Systolic blood pressure (Bp): The upper level of blood pressure scale refers to the amount of pressure in your arteries as your heart muscle shrinks.

Cholesterol (Cl): Cholesterol is a fat found in all the cells of the body.

Diabetes (Db): Diabetes is a medical illness that takes place when your blood glucose, also known as blood 
sugar, is high.

Body mass index (BMI): It is an individual's weight in $\mathrm{kg}$ divided by the square of height in meters. A large value of body mass index (BMI) indicates the quantity of fat in the human body.

Depression (Dp): Depression is a usual and significant disease that adversely affects how you feel, perceive, or behave.

Unhealthy diet (UD): Unhealthy diet means overeating foods that contain a large portion of fat or greasy substances.

Metabolic disorder (MD): A metabolic disorder can occur when unusual chemical reactions in the body change the normal metabolic procedure.

Physical inactivity (PI): PI is a term used to points out people who do not get the suggested level of daily physical activity.

Pre-eclampsia (PC): Pre-eclampsia is a pregnancy problem that occurs when blood pressure is high and it can harm other organs like the liver and kidneys.

Rheumatoid arthritis (RA): Rheumatoid arthritis (RA) is the most ordinary type of autoimmune arthritis. It results when the immune system is not functioning perfectly. RA causes pain and swelling in joints of the body, especially hands and feet.

Rubella (Rb): It is called German measles. Normally, the infected person suffers from this illness for three days.

Heart defect (HD): It is a heart problem causes due to the defect found in the formation of the heart. This defect found at the time of birth.

Thyroids (Th): The thyroid is a butterfly-shaped gland that appears below the front of the neck. Your thyroid lies below your Adam's apple, along the front of the windpipe.

Sleep apnea (SA): Sleep apnea is a serious illness experienced while sleeping. In this problem, patient experience breathing disorders.

Atrial fibrillation (ArF): Atrial fibrillation (AF) is an illness experienced when the heart rate is irregular. Suddenly, the heart beats so fast or too slow, which means irregularly observe in heartbeats.

Homocysteine level (Hm): Homocysteine level is a regular amino acid in human blood. It commonly produces form eating meat. If its level increases from the normal range, then the patient would be most likely to suffer from heart disease.

Pericardial cysts (Pc): Pericardial cysts are an unusual benign congenital anomaly in the middle mediastinum.

Marfan syndrome (MFS): Marfan syndrome (MFS) is a genetic disorder of the connective tissue.

Syphilis inflammation (Sy): Syphilis is a sexually transmitted disease (STD) caused by a type of bacteria called Treponema pallidum. 
Electrolyte imbalance (EI): Calcium, magnesium, potassium, and sodium are called electrolytes. Their disturbing proportion can cause a variety of problems, especially heart problems.

\subsection{Cardiovascular diseases (CVDs) [28]-[31]:}

Coronary artery disease (D1): Coronary artery disease is a narrowing or obstruction of the coronary arteries, most commonly caused by atherosclerosis. Atherosclerosis (sometimes called "stiffness" or "clogging" of the arteries) is the buildup of cholesterol and fat accumulation (called plaque) on the inner walls of the arteries. These plaques can restrict blood flow to the heart muscle either physically by closing the artery or because of abnormal artery tone and functions.

Cardiomyopathy (D2)- Cardiomyopathy is a disease of the heart muscle that makes it difficult for the heart to pump blood to the rest of the body. Cardiomyopathy can cause heart failure. The main types of cardiomyopathy include chronic, hypertrophic, and restrictive cardiomyopathy.

Congenital heart disease (D3)-Congenital heart failure is a problem with the structure of the heart. There are common types of congenital defects. Defects can include walls of the heart, valves of the heart and arteries and veins near the heart.

Heart Attack (D4)- A heart attack occurs when the blood and oxygen supply to the heart is blocked. Fat reserves form plaques in the arteries of the heart over time. If the plaque bursts, the blood clot can form and block the arteries, causing a heart attack.

Heart arrhythmia (D5)- A heart arrhythmia also called an arrhythmia - means your heart is beating too fast, too slow, or with an irregular pattern. Your heart has an electrical system that tells it when to beat and pump blood around your body. If there is a problem with this system you may experience an abnormal heart rhythm.

Peripheral artery disease (D6)- Peripheral artery disease (also known as peripheral artery disease) is a common circulation problem in which narrow arteries reduce blood flow to the organs. When the body suffered from peripheral artery disease (PAD), then the spheres - usually the feet - do not get enough blood flow to keep up with demand.

Aortic disease (D7)- A person with Marfan Syndrome or some related disorder may have an aortic aneurysm (aortic aneurysm) or an aortic aneurysm. These are very serious problems because of the risk of a significantly enlarged aortic tearing or rupture (aortic detection).

Pericardial disease (D8)- Pericardial disease, or pericarditis, is the swelling in any layer of the pericardium. The pericardium is a thin tissue sac that surrounds the heart and contains visceral pericardium - an inner layer that envelops the entire heart.

Deep vein thrombosis (D9)- Deep vein thrombosis is a blood clot that forms in the veins deep in the body. Most deep veins are found in the lower leg or thighs from the clot. If the vein is inflamed, this condition is called thrombophlebitis. A deep vein thrombosis breaks loose and can cause serious problems in the lungs, called pulmonary embolism.

Heart valve disease (D10)- Heart valve disease can be congenital. It can also be caused by many causes and conditions in adults, such as infections and other heart conditions. Heart valve problems can include regression. In this case, the valve flaps do not close properly, which causes blood to flow to the back of your heart.

Heart failure (D11)- Heart failure, sometimes known as heart failure, occurs when the heart muscle does not 
pump blood as it should. Some conditions, such as narrow arteries (coronary artery disease) or hypertension in the heart, slowly leave your heart too weak or too tight to effectively fill and pump.

The following matrix shows the binary relation between symptoms and CVDs. This matrix will be helpful to check the accuracy of the final highlighted cardiovascular disease.

$$
\begin{array}{cccccccccccc}
D 1 & D 2 & D 3 & D 4 & D 5 & D 6 & D 7 & D 8 & D 9 & D 10 & D 11 & \\
1 & 0 & 0 & 1 & 0 & 0 & 1 & 0 & 0 & 0 & 0 & S 1 \\
1 & 0 & 0 & 1 & 1 & 1 & 1 & 0 & 1 & 1 & 0 & S 2 \\
1 & 1 & 1 & 1 & 1 & 0 & 1 & 0 & 1 & 1 & 0 & S 3 \\
1 & 0 & 1 & 1 & 1 & 1 & 1 & 0 & 1 & 0 & 0 & S 4 \\
1 & 1 & 0 & 1 & 1 & 1 & 1 & 0 & 0 & 1 & 1 & S 5 \\
1 & 0 & 0 & 1 & 1 & 1 & 1 & 0 & 0 & 1 & 0 & S 6 \\
1 & 1 & 1 & 1 & 1 & 1 & 1 & 0 & 0 & 1 & 1 & S 7 \\
1 & 1 & 0 & 1 & 1 & 1 & 1 & 0 & 1 & 0 & 1 & S 8 \\
1 & 0 & 0 & 1 & 1 & 1 & 0 & 0 & 0 & 0 & 0 & S 9 \\
1 & 0 & 0 & 1 & 1 & 1 & 1 & 0 & 0 & 0 & 0 & S 10 \\
0 & 1 & 0 & 1 & 0 & 0 & 0 & 0 & 0 & 0 & 0 & S 11 \\
1 & 0 & 0 & 1 & 0 & 1 & 1 & 0 & 1 & 0 & 0 & S 12 \\
0 & 0 & 0 & 1 & 0 & 0 & 0 & 0 & 0 & 0 & 0 & S 13 \\
0 & 0 & 0 & 1 & 0 & 0 & 0 & 0 & 0 & 0 & 0 & S 14 \\
0 & 0 & 0 & 0 & 1 & 0 & 0 & 0 & 0 & 0 & 0 & S 15 \\
0 & 1 & 1 & 0 & 0 & 0 & 0 & 0 & 1 & 0 & 0 & S 16 \\
0 & 0 & 1 & 0 & 0 & 0 & 0 & 0 & 0 & 0 & 0 & S 17 \\
0 & 1 & 1 & 1 & 1 & 0 & 0 & 0 & 1 & 0 & 1 & S 18 \\
1 & 0 & 0 & 1 & 0 & 1 & 0 & 0 & 0 & 0 & 1 & S 19 \\
0 & 1 & 1 & 1 & 1 & 0 & 1 & 0 & 0 & 0 & 1 & S 20 \\
0 & 1 & 1 & 0 & 1 & 0 & 1 & 0 & 0 & 1 & 1 & S 21 \\
0 & 0 & 0 & 0 & 1 & 0 & 0 & 1 & 1 & 0 & 0 & S 22 \\
0 & 1 & 0 & 0 & 1 & 0 & 0 & 0 & 0 & 0 & 0 & S 23 \\
0 & 0 & 0 & 0 & 1 & 0 & 0 & 0 & 0 & 0 & 1 & S 24 \\
0 & 1 & 0 & 1 & 0 & 0 & 0 & 0 & 0 & 1 & 1 & S 25 \\
0 & 1 & 0 & 0 & 1 & 1 & 1 & 1 & 1 & 1 & 1 & S 26 \\
0 & 1 & 0 & 0 & 0 & 0 & 1 & 0 & 0 & 0 & 1 & S 27 \\
0 & 0 & 0 & 0 & 0 & 1 & 0 & 0 & 1 & 0 & 0 & S 28 \\
0 & 0 & 0 & 0 & 0 & 0 & 0 & 1 & 0 & 0 & 0 & S 29 \\
0 & 0 & 0 & 0 & 0 & 0 & 1 & 0 & 0 & 0 & 0 & S 30 \\
0 & 0 & 0 & 0 & 0 & 0 & 1 & 0 & 0 & 0 & 0 & S 31 \\
1 & 1 & 0 & 0 & 0 & 0 & 1 & 0 & 1 & 0 & 0 & S 32 \\
1 & 0 & 0 & 0 & 0 & 0 & 0 & 0 & 1 & 1 & 0 & S 33 \\
0 & 1 & 0 & 0 & 0 & 0 & 0 & 0 & 1 & 0 & 0 & S 34 \\
0 & 0 & 0 & 0 & 1 & 0 & 0 & 0 & 0 & 0 & 0 & S 35 \\
& & & & & & & & & & &
\end{array}
$$

\section{Explainable neutrosophic clinical decision-making system for car- diovascular diseases}

In this section, we will see the detailed working of the neutrosophic clinical decision-making system for cardiovascular diseases. We use single-valued neutrosophic sets for decision making. To make this system easy for the medical professional, we use explainable artificial intelligence approaches. We integrate the explanation part in each module of the system. The explanation module explains the working of that specific module. The quality 
of explanation is measure using causability. We design an algorithm and computes its time complexity. A new de-neutrosophication formula is also proposed in this article. Let's move towards the detailed working of each module.

\subsection{Block diagram of the explainable neutrosophic clinical decision-making system}

There are five major modules of the system. The first module of the system is neutrosophication, the second module is a knowledge base, the third module is the inference engine, the fourth module is de-neutrosophication, and the fifth module is explainability. The system inputs thirty-five variables and calculates the risk of each type of cardiovascular disease. There are major eleven types of cardiovascular diseases. Figure 1 shows the block diagram of the proposed system.

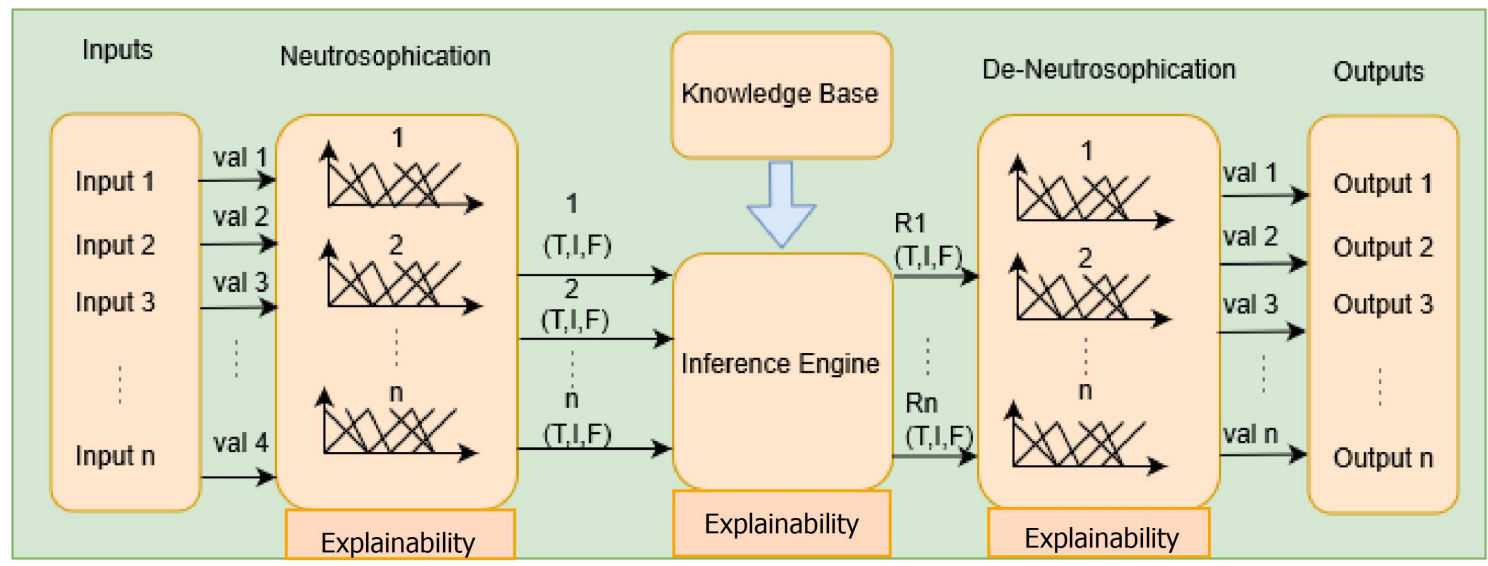

Figure 1: Basic structure of explainable neutrosophic clinical decision-making system

\subsection{General procedure of the system}

Neutrosophic logic (NL) is used as a helping tool for modeling the proposed system. NL is a logic in which each proposition is estimated the percentage of truth in a subset $\mathrm{T}$, the percentage of uncertainty in subset $\mathrm{I}$, and the percentage of error in a subset F, where T, I, F are described above are said to be NL. We conclude that the process of implementing the decision-making system for CVD consists of the following steps:

1- Linguistic variables: Establish system input and output variables.

2- Defining the neutrosophication, inference, and de-neutrosophication mechanisms.

3- Neutrosophication: The process of assigning crisp values to the neutrosophication system as input sets and calculates the degrees of membership, degree of indeterminacy, and degree of falsity of each set.

4- Inference Engine: Apply the NL rules and calculates the output neutrosophic sets concluded from these input sets.

5- De-Neutrosophication: De-neutrosophication is a process of determining the accurate output value from the inferred neutrosophic sets. These crisp values are the final output of the system.

\subsection{Algorithm}

The algorithm of the proposed system is as follows: 


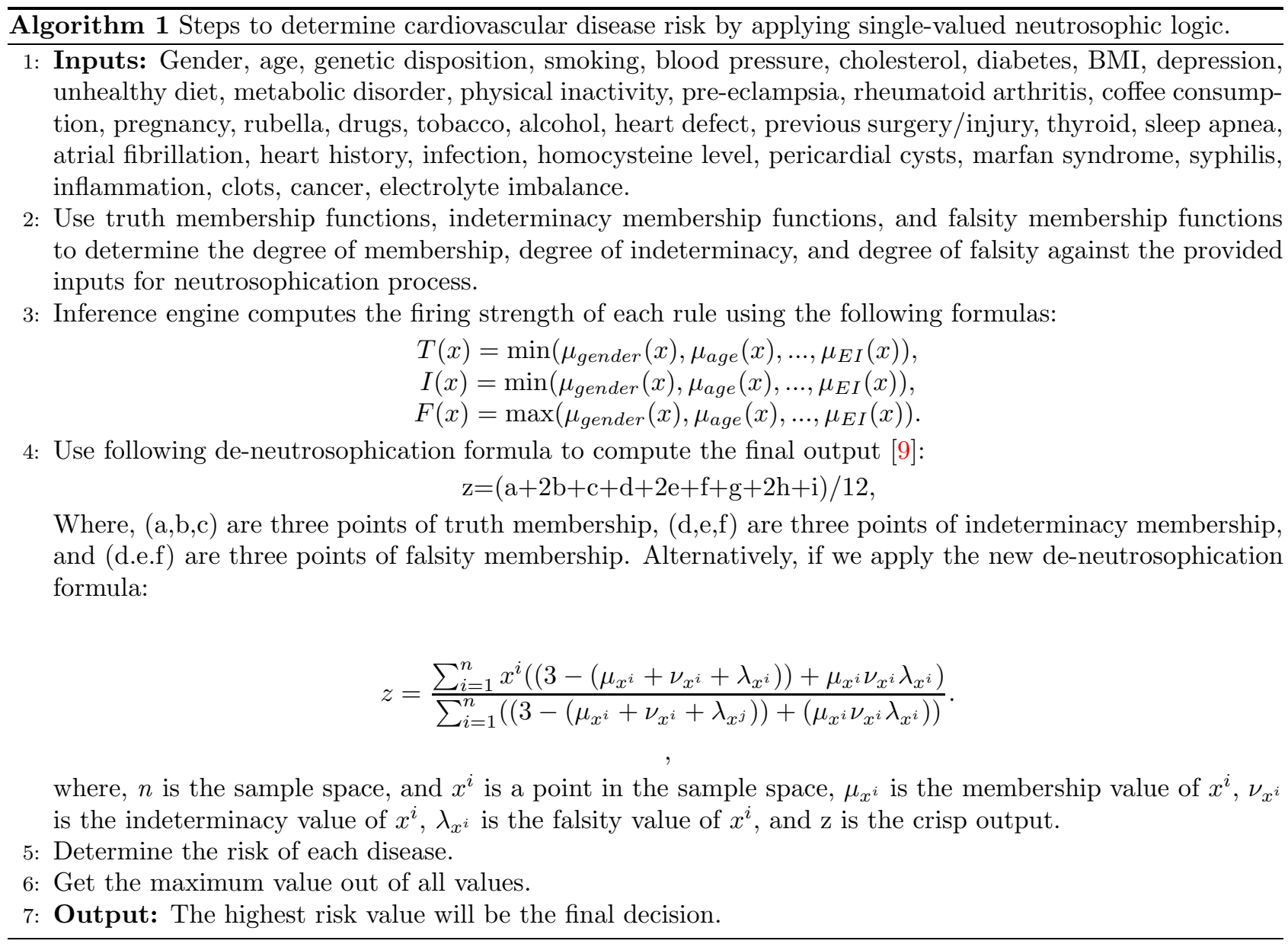

The detail of each supporting algorithm is as follows:

\section{Neutrosophication}

(i). Begin

Time Complexity

(ii). $\quad$ for(i=1 to no. of functions)

(iii).

$\mathrm{DM}[\mathrm{i}]=$ degree of membership of ith membership function

(iv).

$\mathrm{IM}[\mathrm{i}]=$ degree of indeterminacy of ith indeterminacy membership function

(v).

FM[i]=degree of falsity of ith falsity membership function

(vi). $\quad$ end for

(vii). End

Inference Engine

(i). Begin

(ii). $\quad$ for $(\mathrm{i}=1$ to no. of rules $)$

(iii).

$\mathrm{M}[\mathrm{i}]=$ min of degree of membership

(iv).

$\mathrm{I}[\mathrm{i}]=\max$ of degree of indeterminacy

n-1

(v).

$$
\mathrm{F}[\mathrm{i}]=\text { max of degree of falsity }
$$

(vi). end for 


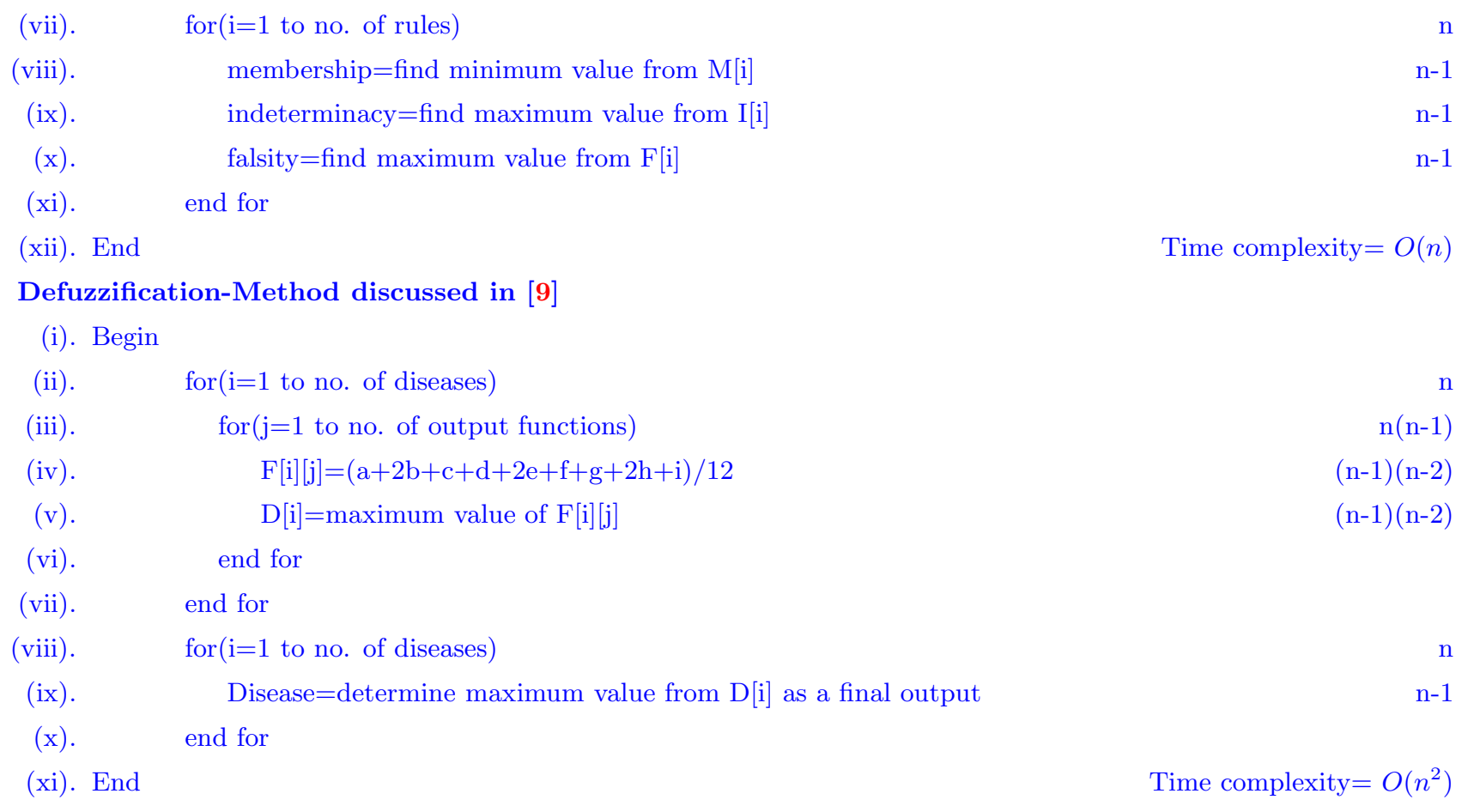

\section{Defuzzification-Proposed method}

(i). Begin

(iii). $\quad$ for $(\mathrm{j}=1$ to no. of point in the functions $) \quad n(n-1)$

(iv). $\quad / /$ jth point along the $\mathrm{x}$-axis in the output membership, indeterminacy, and falsity functions plots

(v). $\quad \mathrm{D}[\mathrm{i}]=\operatorname{Sum}+\frac{x_{j}\left(\left(3-\left(\mu_{x_{j}}+\nu_{x_{j}}+\lambda_{x_{j}}\right)\right)+\left(\mu_{x_{j}} \nu_{x_{j}} \lambda_{x_{j}}\right)\right)}{\left(3-\left(\mu_{x_{j}}+\nu_{x_{j}}+\lambda_{x_{j}}\right)\right)+\left(\mu_{x_{j}} \nu_{x_{j}} \lambda_{x_{j}}\right)} \quad$ (n-1)(n-2)

(vi). $\quad$ end for

(vii). $\quad$ end for

(viii). $\quad$ for $(\mathrm{i}=1$ to no. of diseases) n

(ix). Disease=determine maximum value from D[i] as a final output n-1

(x). $\quad$ end for

(xi). End

Time complexity $=O\left(n^{2}\right)$

Now, we compute the time complexity of Algorithm 1. Line 1 takes O(1) time. The neutrosophication process takes $\mathrm{O}(\mathrm{n})$ time, hence, line 2 takes $\mathrm{O}(\mathrm{n})$ time. The inference engine process takes $\mathrm{O}(\mathrm{n})$ time, therefore, line 3 takes $\mathrm{O}(\mathrm{n})$ time. The de-neutrosophication procedure takes $O\left(n^{2}\right)$ time, therefore, line 4 takes $O\left(n^{2}\right)$. Lines 5 , line 6 , and line 7 takes $\mathrm{O}(1)$. Consequently, the net time complexity of Algorithm 1 is $O\left(n^{2}\right)$.

\subsection{Working of explainable neutrosophic clinical decision-making system for CVDs}

The proposed decision-making system of CVDs is taking thirty-five symptoms as inputs and determines the risk of eleven types of CVDs diseases. The working range of each input variable is described in Table 1. 
Table 1: Range of input variables

\begin{tabular}{|c|c|c|}
\hline Symptom ID & Input Parameters & Range \\
\hline S1 & Gender & $1 / 2$ \\
\hline $\mathrm{S} 2$ & Age (year/s) & $\overline{0-110}$ \\
\hline S3 & Genetic Disposition & $1 / 2$ \\
\hline$\overline{\mathrm{S} 4}$ & Smoking & $1 / 2$ \\
\hline S5 & Systolic Blood Pressure (mm Hg) & $90-150$ \\
\hline S6 & Cholesterol (mg/dL) & $100-400$ \\
\hline S7 & Diabetes $(\mathrm{mg} / \mathrm{dL})$ & $68-300$ \\
\hline S8 & Body Mass Index $\left(\mathrm{kg} / \mathrm{m}^{2}\right)$ & $10-40$ \\
\hline S9 & Depression & $0-2$ \\
\hline S10 & Unhealthy Diet & $\overline{0-2}$ \\
\hline S11 & Metabolic disorder & $1 / 2$ \\
\hline S12 & Physical inactivity & $1 / 2$ \\
\hline S13 & Pre-eclampsia & $1 / 2$ \\
\hline S14 & Rheumatoid arthritis & $1 / 2$ \\
\hline S15 & Coffee consumption & $1 / 2$ \\
\hline S16 & $\begin{array}{l}\text { Pregnancy } \\
\end{array}$ & $1 / 2$ \\
\hline S17 & Rubella & $1 / 2$ \\
\hline S18 & Drugs usage & $1 / 2$ \\
\hline S19 & Tobacco & $1 / 2$ \\
\hline $\mathrm{S} 20$ & Alcohol & $1 / 2$ \\
\hline S21 & heart defect & $1 / 2$ \\
\hline S22 & Previous surgery/injury & $1 / 2$ \\
\hline $\mathrm{S} 23$ & Thyroid & $1 / 2$ \\
\hline S24 & Sleep apnea & $1 / 2$ \\
\hline S25 & Atrial fibrillation & $1 / 2$ \\
\hline S26 & Heart history & $1 / 2$ \\
\hline S27 & Infection & $1 / 2$ \\
\hline S28 & Homocysteine level & $\overline{0-2}$ \\
\hline $\mathrm{S} 29$ & Pericardial cysts & $1 / 2$ \\
\hline S30 & Marfan syndrome & $1 / 2$ \\
\hline S31 & Syphilis & $1 / 2$ \\
\hline S32 & inflammation & $1 / 2$ \\
\hline $\begin{array}{l}\text { S33 } \\
\end{array}$ & Clots & $1 / 2$ \\
\hline S34 & Cancer & $1 / 2$ \\
\hline S35 & Electrolyte Imbalance & $1 / 2$ \\
\hline
\end{tabular}

\subsection{Neutrosophication}

The neutrosophication is a process of converting crisp inputs in linguistic terms by determining the membership degree, indeterminacy degree, and falsity degree. The process of neutrosophication is depicted in the following Figure 2. 


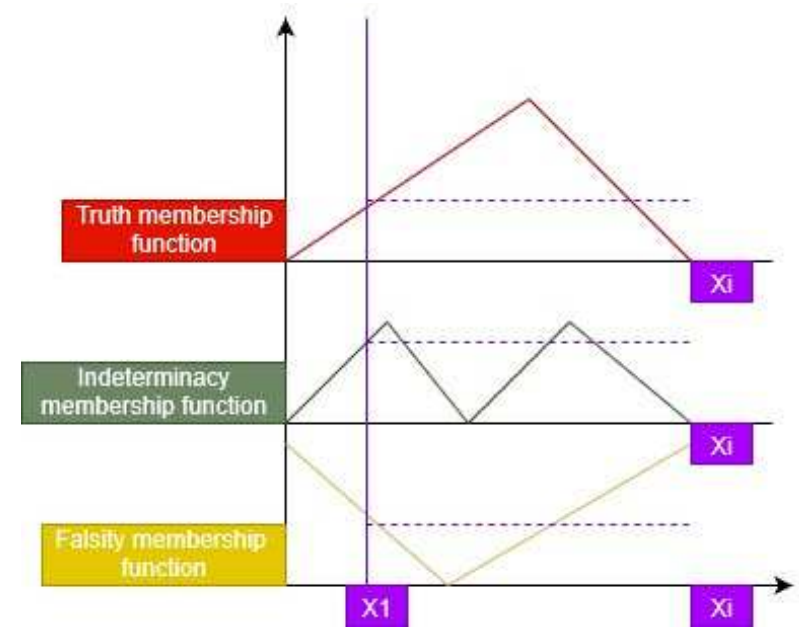

Figure 2: Neutrosophication process [32]

The input range is divided into three types of membership functions. The plot of each input variable is shown in Figure 3, Figure 4, Figure 5, Figure 6, Figure 7, and Figure 8. The green color shows truth membership, yellow color shows indeterminacy membership, and red color show falsity membership.

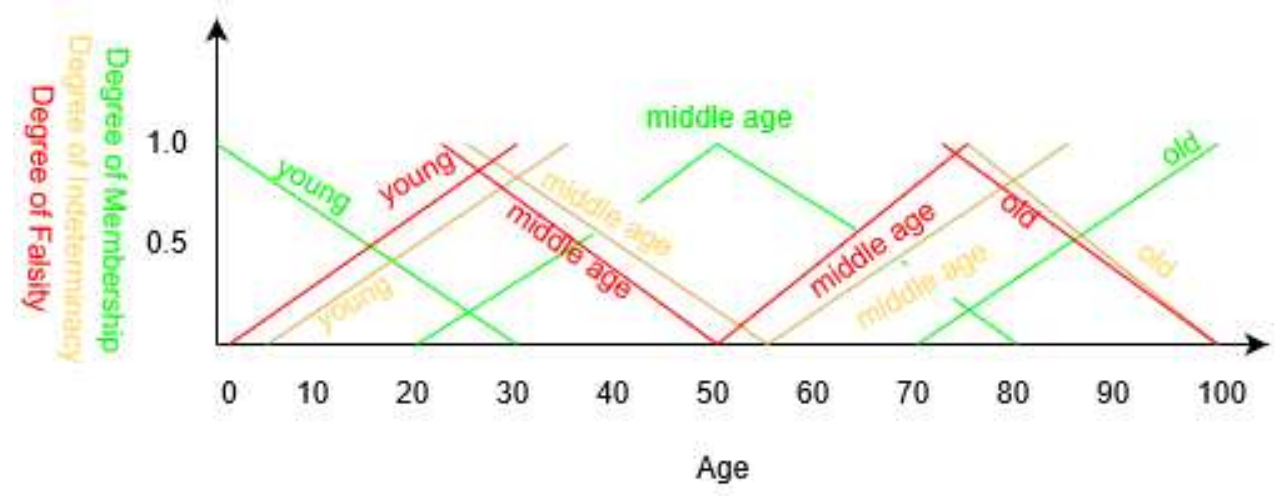

Figure 3: Age 


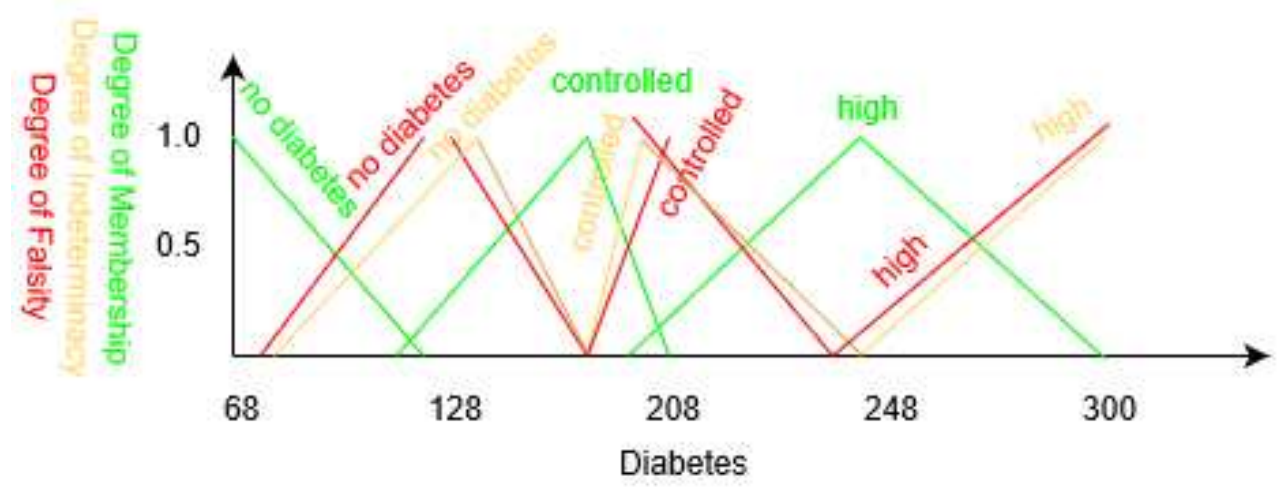

Figure 4: Diabetes

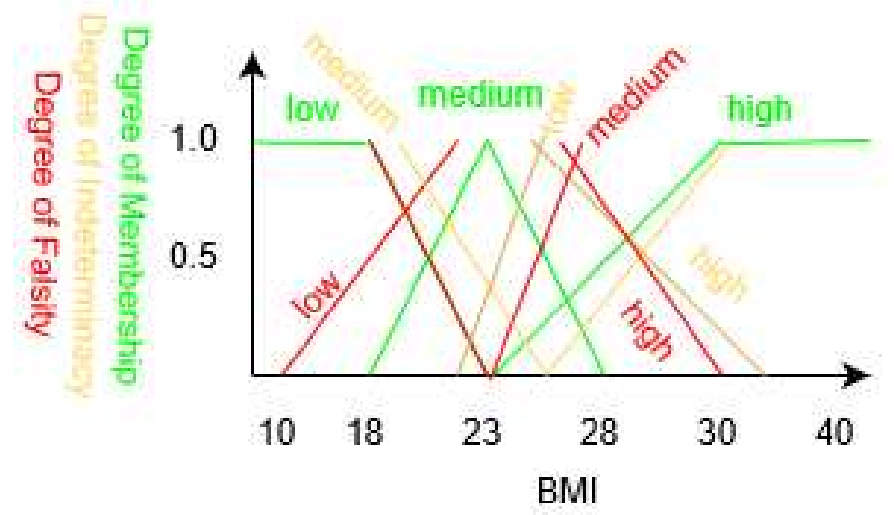

Figure 5: Body mass index (BMI)

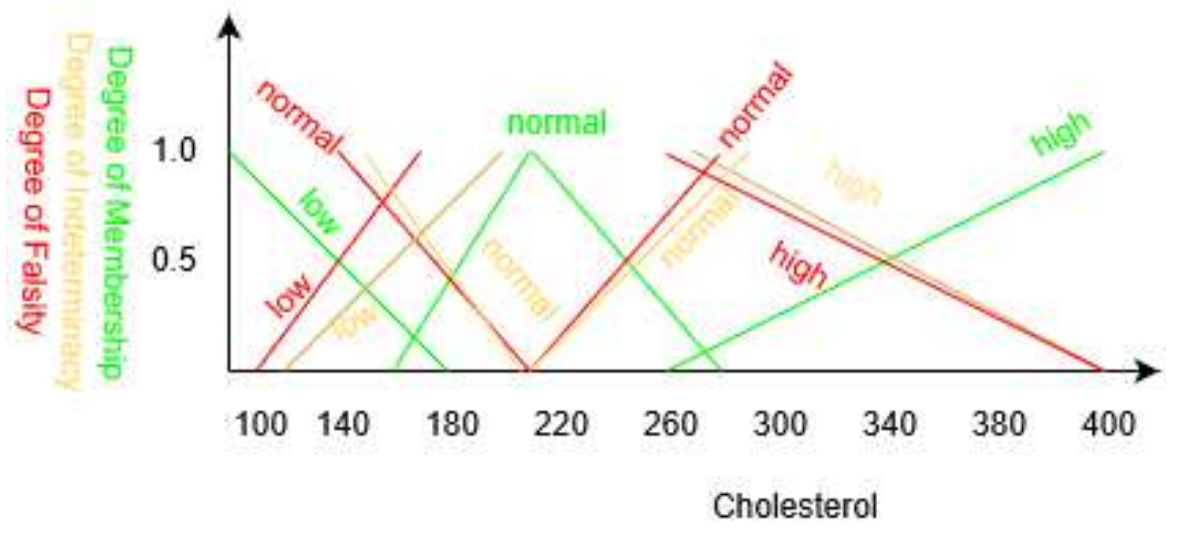

Figure 6: Cholesterol 


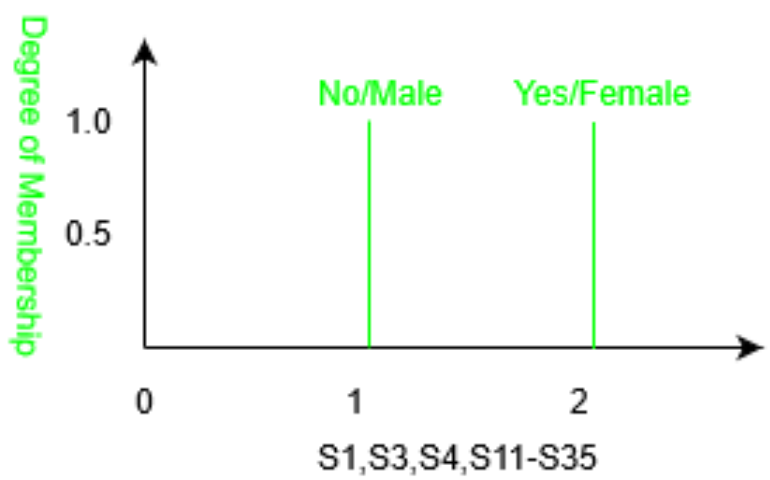

Figure 7: S1,S3,S4,S11-S35

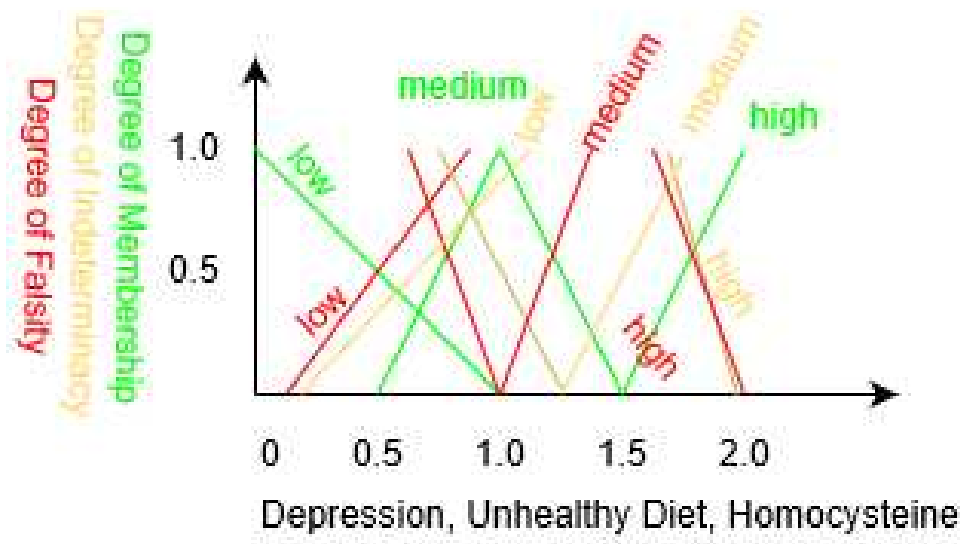

Figure 8: Depression, Unhealthy diet, Homocysteine level

The mathematical form of truth membership, indeterminacy membership, and falsity membership of age are as follows:

$\mu_{\text {young }}(p)=\left\{\begin{array}{ll}\frac{30-p}{30}, & \text { if } p \in[0,30], \\ 0, & \text { otherwise. }\end{array} \quad \mu_{\text {old }}(p)= \begin{cases}\frac{p-70}{30}, & \text { if } p \in[70,100], \\ 0, & \text { otherwise. }\end{cases}\right.$

$\mu_{\text {middle-age }}(p)= \begin{cases}\frac{p-20}{30}, & \text { if } p \in[20,50], \\ \frac{80-p}{30}, & \text { if } p \in[50,80], \\ 0, & \text { otherwise. }\end{cases}$

$\nu_{\text {young }}(p)= \begin{cases}\frac{p-5}{30}, & \text { if } p \in[5,35], \\ 1, & \text { otherwise. }\end{cases}$

$\nu_{\text {old }}(p)= \begin{cases}\frac{100-p}{25}, & \text { if } p \in[75,100] \\ 1, & \text { otherwise }\end{cases}$

$\nu_{\text {middle-age }}(p)= \begin{cases}\frac{55-p}{30}, & \text { if } p \in[25,55], \\ \frac{p-55}{30}, & \text { if } p \in[55,85], \\ 1, & \text { otherwise. }\end{cases}$ 


$$
\begin{array}{ll}
\lambda_{\text {young }}(p)= \begin{cases}\frac{p-3}{27}, & \text { if } p \in[3,30], \\
1, & \text { otherwise. }\end{cases} & \lambda_{\text {old }}(p)= \begin{cases}\frac{100-p}{27}, & \text { if } p \in[73,100], \\
1, & \text { otherwise. }\end{cases} \\
\lambda_{\text {middle-age }}(p)= \begin{cases}\frac{50-p}{27}, & \text { if } p \in[23,50], \\
\frac{p-50}{25}, & \text { if } p \in[50,75], \\
1, & \text { otherwise. }\end{cases}
\end{array}
$$

The mathematical form of degree of membership, degree of indeterminacy, and degree of falsity of blood pressure are as follows:

$$
\begin{aligned}
& \mu_{\text {low }}(p)=\left\{\begin{array}{ll}
\frac{110-p}{20}, & \text { if } p \in[90,110], \\
0, & \text { otherwise. }
\end{array} \quad \mu_{\text {high }}(p)= \begin{cases}\frac{p-135}{10}, & \text { if } p \in[135,145], \\
0, & \text { otherwise. }\end{cases} \right. \\
& \mu_{\text {medium }}(p)= \begin{cases}\frac{p-95}{25}, & \text { if } p \in[90,120] \\
\frac{138-p}{18}, & \text { if } p \in[120,138] \\
0, & \text { otherwise. }\end{cases} \\
& \nu_{\text {low }}(p)= \begin{cases}\frac{p-95}{20}, & \text { if } p \in[95,115] \\
1, & \text { otherwise. }\end{cases} \\
& \nu_{\text {medium }}(p)= \begin{cases}\frac{125-p}{27}, & \text { if } p \in[98,125] \\
\frac{p-125}{17}, & \text { if } p \in[125,142] \\
1, & \text { otherwise. }\end{cases} \\
& \lambda_{\text {low }}(p)= \begin{cases}\frac{p-92}{16}, & \text { if } p \in[92,108] \\
1, & \text { otherwise. }\end{cases} \\
& \lambda_{\text {medium }}(p)= \begin{cases}\frac{120-p}{23}, & \text { if } p \in[97,120], \\
\frac{p-120}{15}, & \text { if } p \in[120,135], \\
1, & \text { otherwise. }\end{cases} \\
& \nu_{\text {high }}(p)= \begin{cases}\frac{145-p}{5}, & \text { if } p \in[140,145] \\
1, & \text { if } p \in[145,150] \\
1, & \text { otherwise. }\end{cases} \\
& \lambda_{\text {high }}(p)= \begin{cases}\frac{145-p}{7}, & \text { if } p \in[138,145] \\
1, & \text { otherwise. }\end{cases}
\end{aligned}
$$

The mathematical form of degree of membership, degree of indeterminacy, and degree of falsity of cholesterol are as follows:

$$
\begin{aligned}
& \mu_{\text {low }}(p)=\left\{\begin{array}{ll}
\frac{180-p}{80}, & \text { if } p \in[100,180], \\
0, & \text { otherwise. }
\end{array} \quad \mu_{\text {high }}(p)= \begin{cases}\frac{p-260}{140}, & \text { if } p \in[260,400], \\
0, & \text { otherwise. }\end{cases} \right. \\
& \mu_{\text {medium }}(p)= \begin{cases}\frac{p-160}{40}, & \text { if } p \in[160,200], \\
\frac{280-p}{80}, & \text { if } p \in[200,280], \\
0, & \text { otherwise. }\end{cases} \\
& \nu_{\text {low }}(p)=\left\{\begin{array}{ll}
\frac{p-105}{80}, & \text { if } p \in[105,185], \\
1, & \text { otherwise. }
\end{array} \quad \nu_{\text {high }}(p)= \begin{cases}\frac{400-p}{135}, & \text { if } p \in[265,400], \\
1, & \text { otherwise. }\end{cases} \right. \\
& \nu_{\text {medium }}(p)= \begin{cases}\frac{205-p}{40}, & \text { if } p \in[165,205], \\
\frac{p-205}{80}, & \text { if } p \in[205,285] \\
1, & \text { otherwise. }\end{cases} \\
& \lambda_{\text {low }}(p)=\left\{\begin{array}{ll}
\frac{p-103}{72}, & \text { if } p \in[103,175], \\
1, & \text { otherwise. }
\end{array} \quad \lambda_{\text {high }}(p)= \begin{cases}\frac{400-p}{138}, & \text { if } p \in[262,400], \\
1, & \text { otherwise. }\end{cases} \right. \\
& \lambda_{\text {medium }}(p)= \begin{cases}\frac{200-p}{37}, & \text { if } p \in[163,200], \\
\frac{p-200}{75}, & \text { if } p \in[200,275], \\
1, & \text { otherwise. }\end{cases}
\end{aligned}
$$

The mathematical form of degree of membership, degree of indeterminacy, and degree of falsity of diabetes are as follows: 


$$
\begin{array}{ll}
\mu_{\text {No-diabetes }}(p)= \begin{cases}\frac{126-p}{58}, & \text { if } p \in[68,126], \\
0, & \text { otherwise. }\end{cases} & \text { if } p \in[180,240], \\
\mu_{\text {medium }}(p)= \begin{cases}\frac{p-125}{45}, & \text { if } p \in[125,170], \\
\frac{200-p}{30}, & \text { if } p \in[170,200], \\
0, & \text { otherwise. }\end{cases} & \mu_{h i g h}(p)= \begin{cases}\frac{p-180}{60}, & \text { if } p \in[240,300], \\
\frac{300-p}{60}, & \text { otherwise. } \\
0, & \text { if } p \in[245,300],\end{cases} \\
\nu_{\text {low }}(p)= \begin{cases}\frac{p-73}{57}, \text { if } p \in[73,130], \\
1, \quad \text { otherwise. }\end{cases} & \text { if } p \in[185,245], \\
\nu_{\text {medium }}(p)= \begin{cases}\frac{175-p}{45}, & \text { if } p \in[130,175], \\
\frac{p-175}{30}, & \text { if } p \in[175,205], \\
1, & \text { otherwise. }\end{cases} & \text { otherwise. }
\end{array}
$$

The mathematical form of degree of membership, degree of indeterminacy, and degree of falsity of BMI are as follows:

$$
\begin{aligned}
& \mu_{\text {low }}(p)= \begin{cases}1, & \text { if } p \in[10,18], \\
\frac{23-p}{5}, & \text { if } p \in[18,23], \\
0, & \text { otherwise. }\end{cases} \\
& \mu_{\text {medium }}(p)=\left\{\begin{array}{ll}
\frac{p-18}{5}, & \text { if } p \in[18,23], \\
\frac{28-p}{5}, & \text { if } p \in[23,28], \\
0, & \text { otherwise. }
\end{array} \quad \mu_{\text {high }}(p)= \begin{cases}\frac{p-23}{7}, & \text { if } p \in[23,30], \\
1, & \text { if } p \in[30,40], \\
0, & \text { otherwise. }\end{cases} \right.
\end{aligned}
$$$$
\nu_{\text {low }}(p)= \begin{cases}0, & \text { if } p \in[10,22] \\ \frac{p-22}{3}, & \text { if } p \in[22,25] \\ 1, & \text { otherwise. }\end{cases}
$$$$
\nu_{\text {medium }}(p)= \begin{cases}\frac{26-p}{4}, & \text { if } p \in[22,26], \\ \frac{p-26}{4}, & \text { if } p \in[26,30], \\ 1, & \text { otherwise. }\end{cases}
$$$$
\nu_{\text {high }}(p)= \begin{cases}0, & \text { if } p \in[33,40] \\ \frac{33-p}{7}, & \text { if } p \in[26,33] \\ 1, & \text { otherwise. }\end{cases}
$$$$
\lambda_{\text {low }}(p)= \begin{cases}0, & \text { if } p \in[10,18] \\ \frac{p-18}{2}, & \text { if } p \in[18,20] \\ 0, & \text { otherwise. }\end{cases}
$$$$
\lambda_{\text {medium }}(p)= \begin{cases}\frac{23-p}{2}, & \text { if } p \in[20,23], \\ \frac{p-23}{2}, & \text { if } p \in[23,25], \\ 1, & \text { otherwise. }\end{cases}
$$

$$
\lambda_{\text {high }}(p)= \begin{cases}\frac{30-p}{5}, & \text { if } p \in[25,30], \\ 0, & \text { if } p \in[30,40] \\ 1, & \text { otherwise. }\end{cases}
$$

The mathematical form of degree of membership, degree of indeterminacy, and degree of falsity of depression, unhealthy diet, and homocysteine level are as follows: 


$$
\begin{aligned}
& \mu_{\text {low }}(p)=\left\{\begin{array}{ll}
1-p, & \text { if } p \in[0,1], \\
0, & \text { otherwise. }
\end{array} \quad \mu_{\text {high }}(p)= \begin{cases}\frac{p-1.5}{0.5}, & \text { if } p \in[1.5,2], \\
0, & \text { otherwise. }\end{cases} \right. \\
& \mu_{\text {medium }}(p)= \begin{cases}\frac{p-0.5}{0.5}, & \text { if } p \in[0.5,1] \\
\frac{1.5-p}{0.5}, & \text { if } p \in[1,1.5] \\
0, & \text { otherwise. }\end{cases} \\
& \nu_{\text {low }}(p)=\left\{\begin{array}{ll}
p-0.2, & \text { if } p \in[0.2,1.2], \\
1, & \text { otherwise. }
\end{array} \quad \nu_{\text {high }}(p)= \begin{cases}\frac{2-p}{0.3}, & \text { if } p \in[1.7,2] \\
1, & \text { otherwise }\end{cases} \right. \\
& \nu_{\text {medium }}(p)= \begin{cases}\frac{1.2-p}{0.5}, & \text { if } p \in[0.7,1.2] \\
\frac{p-1.2}{0.6}, & \text { if } p \in[1.2,1.8] \\
1, & \text { otherwise }\end{cases} \\
& \lambda_{\text {low }}(p)= \begin{cases}\frac{p-0.1}{0.8}, & \text { if } p \in[0.1,0.9], \\
1, & \text { otherwise. }\end{cases} \\
& \lambda_{\text {medium }}(p)= \begin{cases}\frac{1-p}{0.4}, & \text { if } p \in[0.6,1] \\
\frac{p-1}{0.4}, & \text { if } p \in[1,1.4] \\
1, & \text { otherwise. }\end{cases} \\
& \lambda_{\text {high }}(p)= \begin{cases}\frac{2-p}{0.4}, & \text { if } p \in[1.6,2] \\
1, & \text { otherwise }\end{cases}
\end{aligned}
$$

The mathematical form of degree of membership, degree of indeterminacy, and degree of falsity of S1,S3, S4 and S11 to S35 are as follows:

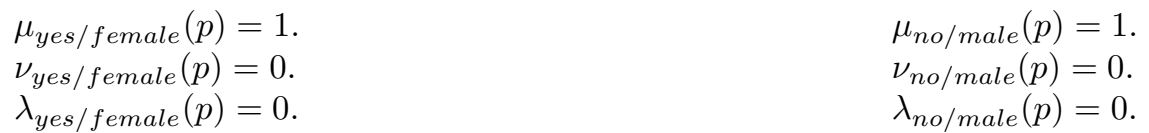

\subsubsection{Ante-hoc Explanation}

In this module, we define linguistic variables of all inputs and their truth membership, indeterminacy membership, and falsity membership. We have written their mathematical forms and represented them using their plots. These membership functions enable us to determine how much an input value is true, uncertain, and false. The value of these functions lies between 0 to 1 . The value of each linguistic variable is the output of the neutrosophication module.

\subsection{Inference Engine}

The inference engine is the major component of the decision-making systems that applies logical rules to knowledge-based to get new information. It contains human-like judgments which are expressed by the rules. Rules are IF-THEN statements. There are 665 rules of our system. Some of them are described below:

R1 - IF (gender $=$ male, age $=$ middle-age, genetic disposition=yes, smoking=yes, blood pressure $=$ high, cholesterol=high, diabetes $=$ high, $\mathrm{BMI}=$ medium, depression=high, unhealthy diet=frequent, metabolic disorder $=$ no, physical inactivity $=$ yes, pre-eclampsia $=$ no, rheumatoid arthritis $=$ no, coffee consumption $=$ no, pregnancy $=$ no, rubella $=$ no, drugs $=$ no, tobacco $=$ yes, alcohol $=$ no, heart defect $=$ no, previous surgery $/$ injury $=$ no, thyroid $=$ no, sleep apnea $=$ no, atrial fibrillation $=$ no, heart history $=$ no, infection $=$ no, homocysteine level $=$ low, pericardial cysts $=$ no, marfan syndrome $=$ no, syphilis $=$ no, inflammation $=y e s$, clots $=$ yes, cancer $=$ no, electrolyte imbalance=no ) THEN (coronary artery disease=very high, cardiomyopathy=low, congenital heart disease=low, heart attack=Below medium, heart arrhythmia=low, peripheral artery disease=below medium, aorta disease $=$ low, and marfan syndrome $=$ low, pericardial disease $=$ low, deep vein thrombosis $=$ low, heart valve disease $=$ low, heart failure $=$ low).

R2 - IF (gender=male, age =middle-age, genetic disposition=yes, smoking=yes, blood pressure=high, cholesterol=high, diabetes=high, $\mathrm{BMI}=$ high, depression=high, unhealthy diet=frequent, metabolic disorder=no, 
physical inactivity $=$ yes, pre-eclampsia $=$ no, rheumatoid arthritis $=$ no, coffee consumption $=$ no, pregnancy $=$ no, rubella $=$ no, drugs $=$ no, tobacco $=$ yes, alcohol $=$ no, heart defect $=$ no, previous surgery $/$ injury $=$ no, thyroid $=$ no, sleep apnea $=$ no, atrial fibrillation $=$ no, heart history $=$ no, infection $=$ no, homocysteine level=low, pericardial cysts $=$ no, marfan syndrome $=$ no, syphilis $=$ no, inflammation $=y e s$, clots=yes, cancer $=$ no, electrolyte imbalance $=$ no ) THEN (coronary artery disease=very high, cardiomyopathy=low, Congenital heart disease=low, heart attack=below medium, heart arrhythmia $=$ low, peripheral artery disease=below medium, aorta disease=low, and marfan syndrome $=$ low, pericardial disease $=$ low, deep vein thrombosis $=$ low, heart valve disease $=$ low, heart failure=low).

R3 - IF (gender=male OR female, age=middle-age OR old, genetic disposition=yes, smoking=no, blood pressure $=$ medium, cholesterol $=$ low, diabetes $=$ medium, $B M I=$ medium, depression $=$ low, unhealthy diet $=$ low, metabolic disorder $=$ no, physical inactivity $=$ no, pre-eclampsia $=$ no, rheumatoid arthritis $=$ no, coffee consumption $=$ no, pregnancy $=$ no OR yes, rubella $=$ no, drugs $=$ yes, tobacco $=$ no, alcohol $=$ yes, heart defect $=$ no, previous surgery/injury $=$ no, thyroid $=$ no, sleep apnea $=$ no, atrial fibrillation=yes, heart history=yes, infection $=$ yes, homocysteine level=low, pericardial cysts $=$ no, marfan syndrome $=$ no, syphilis $=$ no, inflammation $=$ yes, $c$ lots $=$ yes, cancer $=$ yes, electrolyte imbalance $=$ no $)$ THEN (coronary artery disease $=$ low, cardiomyopathy $=$ medium, congenital heart disease $=$ low, heart attack=low, heart arrhythmia=below medium, peripheral artery disease=low, aorta disease $=$ low, and marfan syndrome $=$ low, pericardial disease $=$ low, deep vein thrombosis=low, heart valve disease $=$ low, heart failure $=$ medium).

R4-IF (gender $=$ male OR female, age=middle-age OR old, genetic disposition=yes, smoking=no, blood pressure $=$ high, cholesterol $=$ low, diabetes $=$ high, $\mathrm{BMI}=$ medium, depression=low, unhealthy diet=low, metabolic disorder $=$ no, physical inactivity $=$ no, pre-eclampsia $=$ no, rheumatoid arthritis $=$ no, coffee consumption $=$ no, pregnancy $=$ no OR yes, rubella $=$ no, drugs $=$ yes, tobacco $=$ no, alcohol $=$ yes, heart defect $=$ no, previous surgery $/$ injury $=$ no, thyroid $=$ no, sleep apnea $=$ no, atrial fibrillation $=$ yes, heart history $=$ yes, infection=yes, homocysteine level $=$ low, pericardial cysts $=$ no, marfan syndrome $=$ no, syphilis $=$ no, inflammation $=$ yes, clots $=$ yes, cancer $=$ yes, electrolyte imbalance=no ) THEN (coronary artery disease=low, cardiomyopathy=very high, congenital heart disease $=$ low, heart attack=low, heart arrhythmia=below medium, peripheral artery disease $=$ low, aorta disease $=$ low, and marfan syndrome $=$ low, pericardial disease $=$ low, deep vein thrombosis $=$ low, heart valve disease $=$ low, heart failure $=$ medium).

R5 - IF (gender $=$ male, age $=$ young, genetic disposition $=$ yes, smoking $=$ yes, blood pressure $=$ low, cholesterol $=$ low, diabetes $=$ high, $\mathrm{BMI}=$ medium, depression $=$ low, unhealthy diet $=$ low, metabolic disorder $=$ no, physical inactivity $=$ no, pre-eclampsia $=$ no, rheumatoid arthritis $=$ no, coffee consumption $=$ no, pregnancy $=$ no, rubella $=$ yes, drugs $=$ yes, tobacco $=$ no, alcohol $=$ yes, heart defect $=$ no, previous surgery $/$ injury $=$ no, thyroid $=$ no, sleep apnea $=$ no, atrial fibrillation $=$ no, heart history $=$ no, infection $=$ no, homocysteine level $=$ low, pericardial cysts $=$ no, marfan syndrome $=$ no, syphilis $=$ no, inflammation $=$ no, clots $=$ no, cancer $=$ yes, electrolyte imbalance $=$ no ) THEN (coronary artery disease $=$ low, cardiomyopathy $=$ low, congenital heart disease $=$ high, heart attack $=$ low, heart arrhythmia $=$ low, peripheral artery disease $=$ low, aorta disease $=$ low, and marfan syndrome $=$ low, pericardial disease $=$ low, deep vein thrombosis $=$ low, heart valve disease $=$ low, heart failure $=$ low).

R6 $-\mathbf{I F}$ (gender $=$ male OR female, age $=$ old, genetic disposition $=$ yes, smoking $=$ yes, blood pressure $=$ medium, cholesterol $=$ medium, diabetes $=$ medium, $\mathrm{BMI}=$ medium, depression $=$ medium, unhealthy diet $=$ medium, metabolic disorder $=$ no, physical inactivity $=$ yes, pre-eclampsia $=$ no, rheumatoid arthritis $=$ no, coffee consumption $=$ no, pregnancy $=$ no, rubella $=$ no, drugs $=$ no, tobacco $=$ yes, alcohol $=$ no, heart defect $=$ no, previous surgery $/$ injury $=$ no, thyroid $=$ no, sleep apnea $=$ no, atrial fibrillation $=$ no, heart history $=$ no, infection $=$ no, homocysteine level $=$ medium, pericardial cysts $=$ no, $\operatorname{marfan}$ syndrome $=$ no, syphilis $=$ no, inflammation $=$ no, clots $=$ no, cancer $=$ no, electrolyte imbalance $=$ no $)$ THEN (coronary artery disease $=$ medium, cardiomyopathy $=$ low, congenital heart disease $=$ low, heart attack $=$ low, heart arrhythmia $=$ low, peripheral artery disease=very high, aorta disease $=$ low, and marfan syndrome $=$ low, pericardial disease $=$ low, deep vein thrombosis $=$ low, heart valve dis- 
ease $=$ low, heart failure $=$ low $)$.

\subsubsection{Ante-hoc Explanation}

The knowledgebase is a very important part of our system. It contains all possible rules which portray human intelligence. These rules are IF-THEN statements, which represent how to relate inputs with desired outputs. The inference engine takes the linguistic value of the neutrosophication module and determines active rules out of all possible rules. The output of each active rule is simply the minimum value of all membership functions, maximum value of all indeterminacy membership functions, and maximum value of all falsity membership functions which comes under the IF statement of that rule. The value of each active rule is called the firing strength of the rule.

\subsection{De-Neutrosophication}

The last step of our decision-making system is de-neutrosophication. In this paper, we use two ways to perform de-neutrosophication. The first de-neutrosophication method is taken from the literature, as discussed in [9]. The second method is proposed by us and it is the modification of the defuzzification formula discussed in [33]. The modified de-neutrosophication formula is as follows:

$$
z=\frac{\sum_{i=1}^{n} x^{i}\left(\left(3-\left(\mu_{x^{i}}+\nu_{x^{i}}+\lambda_{x^{i}}\right)\right)+\mu_{x^{i}} \nu_{x^{i}} \lambda_{x^{i}}\right)}{\sum_{i=1}^{n}\left(\left(3-\left(\mu_{x^{i}}+\nu_{x^{i}}+\lambda_{x^{j}}\right)\right)+\left(\mu_{x^{i}} \nu_{x^{i}} \lambda_{x^{i}}\right)\right)}
$$

where,

$n$ is the sample space, and $x^{i}$ is a point in the sample space, $\mu_{x^{i}}$ is the membership value of $x^{i}, \nu_{x^{i}}$ is the indeterminacy value of $x^{i}, \lambda_{x^{i}}$ is the falsity value of $x^{i}$, and $\mathrm{z}$ is the crisp output.

The plots of degree of membership, degree of indeterminacy, and degree of falsity for output parameter (i.e. CVD risk) are shown in Figure 9, Figure 10, and Figure 11.

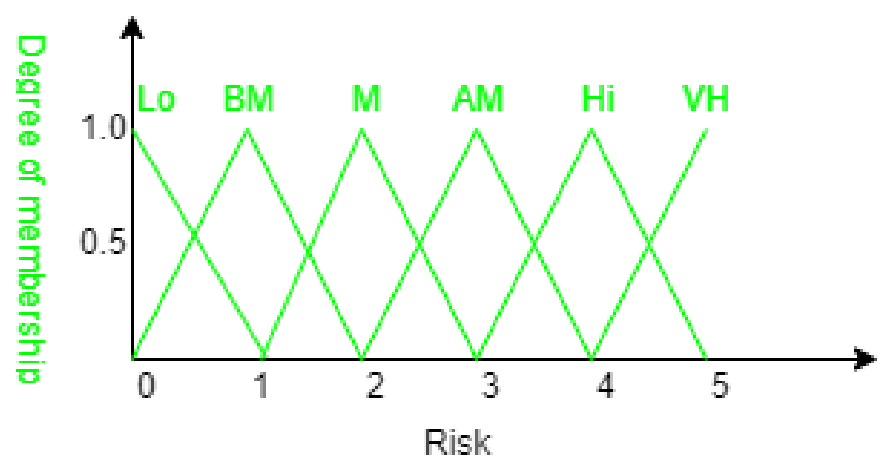

Figure 9: CVD risk-degree of membership 


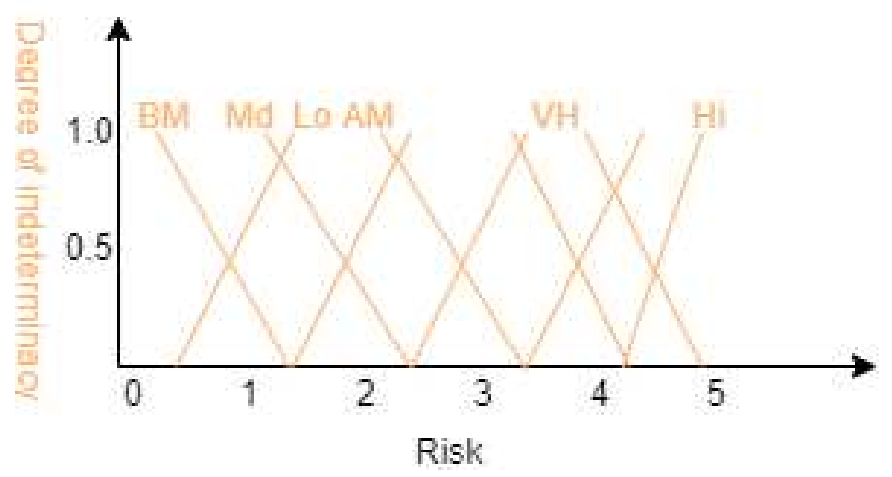

Figure 10: CVD risk-degree of indeterminacy

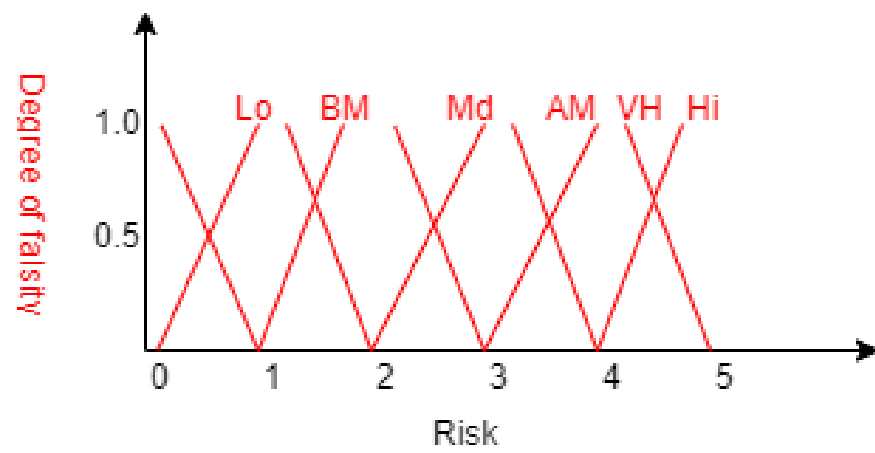

Figure 11: CVD risk-degree of falsity

The mathematical form of truth membership, indeterminacy membership, and falsity membership of CVDs risk are as follows:

$\mu_{\text {low }}(p)=\left\{\begin{array}{ll}1-p, & \text { if } p \in[0,1], \\ 0, & \text { otherwise. }\end{array} \quad \mu_{\text {medium }}(p)= \begin{cases}p, & \text { if } p \in[1,2], \\ 3-p, & \text { if } p \in[2,3], \\ 0, & \text { otherwise. }\end{cases}\right.$

$\mu_{\text {below-medium }}(p)= \begin{cases}p, & \text { if } x \in[0,1] \\ 2-p, & \text { if } x \in[1,2], \\ 0, & \text { otherwise. }\end{cases}$

$\mu_{\text {above-medium }}(p)=\left\{\begin{array}{ll}p-2, & \text { if } p \in[2,3], \\ 4-p, & \text { if } p \in[3,4], \\ 0, & \text { otherwise. }\end{array} \quad \mu_{\text {very-high }}(p)= \begin{cases}p-4, & \text { if } p \in[4,5] \\ 0, & \text { otherwise }\end{cases}\right.$

$\mu_{\text {high }}(p)= \begin{cases}p-3, & \text { if } p \in[3,4] \\ 5-p, & \text { if } p \in[4,5] \\ 0, & \text { otherwise }\end{cases}$ 


$$
\begin{aligned}
& \nu_{\text {low }}(p)= \begin{cases}\frac{p-0.3}{0.9}, & \text { if } p \in[0.3,1.2] \\
1, & \text { otherwise. }\end{cases} \\
& \nu_{\text {below-medium }}(p)= \begin{cases}\frac{1.5-p}{1.3}, & \text { if } p \in[0.2,1.5], \\
p-1.5, & \text { if } p \in[1.5,2.5], \\
1, & \text { otherwise. }\end{cases} \\
& \nu_{\text {medium }}(p)= \begin{cases}2.5-p, & \text { if } p \in[1.5,2.5] \\
p-2.5, & \text { if } p \in[2.5,3.5] \\
1, & \text { otherwise. }\end{cases} \\
& \nu_{\text {above-medium }}(p)= \begin{cases}3.5-p, & \text { if } p \in[2.5,3.5], \\
p-3.5, & \text { if } p \in[3.5,4.5], \\
1, & \text { otherwise. }\end{cases} \\
& \nu_{\text {high }}(p)= \begin{cases}4.5-p, & \text { if } p \in[3.5,4.5] \\
p-4.5, & \text { if } p \in[4.5,5.0] \\
1, & \text { otherwise. }\end{cases} \\
& \nu_{\text {very-high }}(p)= \begin{cases}\frac{5-p}{0.7}, & \text { if } p \in[4.3,5], \\
1, & \text { otherwise. }\end{cases} \\
& \lambda_{\text {below-medium }}(p)= \begin{cases}\frac{1-p}{0.9}, & \text { if } p \in[0.1,1], \\
\frac{p-1}{0.7}, & \text { if } p \in[1,1.7], \\
1, & \text { otherwise. }\end{cases} \\
& \lambda_{\text {above-medium }}(p)= \begin{cases}\frac{3-p}{0.8}, & \text { if } p \in[2.2,3] \\
\frac{p-3}{1.2}, & \text { if } p \in[3,4.2] \\
1, & \text { otherwise. }\end{cases} \\
& \lambda_{\text {very-high }}(p)= \begin{cases}\frac{5-p}{0.8}, & \text { if } p \in[4.2,5] \\
1, & \text { otherwise. }\end{cases} \\
& \lambda_{\text {low }}(p)= \begin{cases}\frac{p-0.2}{0.7}, & \text { if } p \in[0.2,0.9] \\
1, & \text { otherwise. }\end{cases} \\
& \lambda_{\text {medium }}(x)= \begin{cases}\frac{2-p}{0.8}, & \text { if } p \in[1.2,2], \\
\frac{p-2}{1.3}, & \text { if } p \in[2,3.3], \\
1, & \text { otherwise. }\end{cases} \\
& \lambda_{\text {high }}(p)= \begin{cases}\frac{4-p}{0.8}, & \text { if } p \in[3.2,4] \\
\frac{p-4}{0.8}, & \text { if } p \in[4,4.8] \\
1, & \text { otherwise. }\end{cases}
\end{aligned}
$$

\subsubsection{Ante-hoc Explanation}

De-neutrosophication is the last step of our system. This part takes firing strength of the active rules and maps these values on output membership functions of CVDs risk. We mathematically defined the truth membership, indeterminacy membership, and falsity membership of each CVDs and show their plots too. The output of de-neutrosophicaion is a crisp value for each output, which shows the risk of each CVDs. We will take the maximum value of risk as a final output.

\section{Case study}

This section describes an example of a neutrosophic clinical decision-making system as a tool to analyze the risk of each cardiovascular disease. For this purpose, consider an input: (age, gender, genetic disposition, smoking , blood pressure, cholesterol, diabetes, body mass index ,depression, unhealthy diet, metabolic disorder, physical inactivity, pre-eclampsia, rheumatoid arthritis, coffee consumption, pregnancy, rubella, drugs, tobacco, alcohol, heart defect, previous surgery/injury, thyroid, sleep apnea, atrial fibrillation, heart history, infection, homocysteine level, pericardial cysts, marfan syndrome, syphilis, inflammation, clots, cancer, electrolyte imbalance )$=(60,1,2,2,143,290,210,29,1.9,1.75,1,2,1,1,1,1,1,1,2,1,1,1,1,1,1,1,1,0.1,1,1,1,2,2,1$, 1). Now we will pass the above input from each module of the system and show the detailed working of the proposed system.

\subsection{Neutrosophication}

The first module of the neutrosophic clinical decision-making system for CVDs is neutrosophication. After passing the considered input of this module, we get the following output:

Age $(60)=\left(\mu_{\text {young }}, \mu_{\text {middle-age }}, \mu_{\text {old }}\right)=(0,0.67,0),\left(\nu_{\text {young }}, \nu_{\text {middle-age }}, \nu_{\text {old }}\right)=(1,0.17,1),\left(\lambda_{\text {young }}, \lambda_{\text {middle-age }}, \lambda_{\text {old }}\right)=(1,0.4,1)$ 
$\operatorname{Gender}(1)=\left(\mu_{\text {male }}, \mu_{\text {female }}\right)=(1,0),\left(\nu_{\text {male }}, \nu_{\text {female }}\right)=(0,0),\left(\lambda_{\text {male }}, \lambda_{\text {female }}\right)=(0,0)$.

Genetic $\operatorname{disposition}(2)=\left(\mu_{y e s}, \mu_{n o}\right)=(1,0),\left(\nu_{y e s}, \nu_{n o}\right)=(0,0),\left(\lambda_{\text {yes }}, \lambda_{n o}\right)=(0,0)$.

$\operatorname{Smoking}(2)=\left(\mu_{y e s}, \mu_{n o}\right)=(1,0),\left(\nu_{y e s}, \nu_{n o}\right)=(0,0),\left(\lambda_{\text {yes }}, \lambda_{\text {no }}\right)=(0,0)$.

Blood pressure $(143)=\left(\mu_{\text {low }}, \mu_{\text {medium }}, \mu_{\text {high }}\right)=(0,0,0.8),\left(\nu_{\text {low }}, \nu_{\text {medium }}, \nu_{\text {high }}\right)=(1,1,0.4),\left(\lambda_{\text {low }}, \lambda_{\text {medium }}, \lambda_{\text {high }}\right)=(1,1,0.29)$.

Cholesterol $(290)=\left(\mu_{\text {low }}, \mu_{\text {medium }}, \mu_{\text {high }}\right)=(0,0,0.21),\left(\nu_{\text {low }}, \nu_{\text {medium }}, \nu_{\text {high }}\right)=(1,1,0.81),\left(\lambda_{\text {low }}, \lambda_{\text {medium }}\right.$, $\left.\lambda_{\text {high }}\right)=(1,1,0.8)$.

$\operatorname{Diabetes}(210)=\left(\mu_{\text {low }}, \mu_{\text {medium }}, \mu_{\text {high }}\right)=(0,0,0.5),\left(\nu_{\text {low }}, \nu_{\text {medium }}, \nu_{\text {high }}\right)=(1,1,0.58),\left(\lambda_{\text {low }}, \lambda_{\text {medium }}, \lambda_{\text {high }}\right)=$ $(1,1,0.53)$.

$\operatorname{BMI}(29)=\left(\mu_{\text {low }}, \mu_{\text {medium }}, \mu_{\text {high }}\right)=(0,0,0.86),\left(\nu_{\text {low }}, \nu_{\text {medium }}, \nu_{\text {high }}\right)=(1,0.75,0.57),\left(\lambda_{\text {low }}, \lambda_{\text {medium }}, \lambda_{\text {high }}\right)=$ $(1,1,0.2)$.

$\operatorname{Depression}(1.9)=\left(\mu_{\text {low }}, \mu_{\text {medium }}, \mu_{\text {high }}\right)=(0,0,0.8),\left(\nu_{\text {low }}, \nu_{\text {medium }}, \nu_{\text {high }}\right)=(1,1,0.33),\left(\lambda_{\text {low }}, \lambda_{\text {medium }}, \lambda_{\text {high }}\right)=(1,1,0.25)$.

Unhealthy $\operatorname{diet}(1.75)=\left(\mu_{\text {low }}, \mu_{\text {medium }}, \mu_{\text {high }}\right)=(0,0,0.5),\left(\nu_{\text {low }}, \nu_{\text {medium }}, \nu_{\text {high }}\right)=(1,1,0.83),\left(\lambda_{\text {low }}, \lambda_{\text {medium }}\right.$, $\left.\lambda_{\text {high }}\right)=(1,1,0.625)$.

Metabolic disorder $(1)=\left(\mu_{\text {yes }}, \mu_{n o}\right)=(0,1),\left(\nu_{\text {yes }}, \nu_{n o}\right)=(0,0),\left(\lambda_{\text {yes }}, \lambda_{n o}\right)=(0,0)$.

Physical inactivity $(2)=\left(\mu_{\text {yes }}, \mu_{n o}\right)=(1,0),\left(\nu_{y e s}, \nu_{n o}\right)=(0,0),\left(\lambda_{\text {yes }}, \lambda_{\text {no }}\right)=(0,0)$.

Pre-eclampsia $(1)=\left(\mu_{\text {yes }}, \mu_{n o}\right)=(0,1),\left(\nu_{\text {yes }}, \nu_{n o}\right)=(0,0),\left(\lambda_{\text {yes }}, \lambda_{n o}\right)=(0,0)$.

Rheumatoid $\operatorname{arthritis}(1)=\left(\mu_{\text {yes }}, \mu_{n o}\right)=(0,1),\left(\nu_{\text {yes }}, \nu_{n o}\right)=(0,0),\left(\lambda_{\text {yes }}, \lambda_{n o}\right)=(0,0)$.

Coffee consumption $(1)=\left(\mu_{y e s}, \mu_{n o}\right)=(0,1),\left(\nu_{y e s}, \nu_{n o}\right)=(0,0),\left(\lambda_{\text {yes }}, \lambda_{n o}\right)=(0,0)$.

Pregnancy $(1)=\left(\mu_{\text {yes }}, \mu_{n o}\right)=(0,1),\left(\nu_{\text {yes }}, \nu_{n o}\right)=(0,0),\left(\lambda_{\text {yes }}, \lambda_{n o}\right)=(0,0)$.

$\operatorname{Rubella}(1)=\left(\mu_{\text {yes }}, \mu_{\text {no }}\right)=(0,1),\left(\nu_{\text {yes }}, \nu_{n o}\right)=(0,0),\left(\lambda_{\text {yes }}, \lambda_{\text {no }}\right)=(0,0)$.

$\operatorname{Drugs}(1)=\left(\mu_{\text {yes }}, \mu_{n o}\right)=(0,1),\left(\nu_{\text {yes }}, \nu_{n o}\right)=(0,0),\left(\lambda_{\text {yes }}, \lambda_{n o}\right)=(0,0)$.

$\operatorname{Tobacco}(2)=\left(\mu_{\text {yes }}, \mu_{n o}\right)=(1,0),\left(\nu_{\text {yes }}, \nu_{n o}\right)=(0,0),\left(\lambda_{\text {yes }}, \lambda_{\text {no }}\right)=(0,0)$.

$\operatorname{Alcohol}(1)=\left(\mu_{\text {yes }}, \mu_{n o}\right)=(0,1),\left(\nu_{\text {yes }}, \nu_{\text {no }}\right)=(0,0),\left(\lambda_{\text {yes }}, \lambda_{\text {no }}\right)=(0,0)$.

heart defect $(1)=\left(\mu_{\text {yes }}, \mu_{n o}\right)=(0,1),\left(\nu_{\text {yes }}, \nu_{n o}\right)=(0,0),\left(\lambda_{\text {yes }}, \lambda_{\text {no }}\right)=(0,0)$.

Previous surgery/injury $(1)=\left(\mu_{\text {yes }}, \mu_{n o}\right)=(0,1),\left(\nu_{\text {yes }}, \nu_{n o}\right)=(0,0),\left(\lambda_{\text {yes }}, \lambda_{n o}\right)=(0,0)$.

$\operatorname{Thyroid}(1)=\left(\mu_{\text {yes }}, \mu_{n o}\right)=(0,1),\left(\nu_{y e s}, \nu_{n o}\right)=(0,0),\left(\lambda_{\text {yes }}, \lambda_{\text {no }}\right)=(0,0)$.

Sleep apnea $(1)=\left(\mu_{\text {yes }}, \mu_{n o}\right)=(0,1),\left(\nu_{\text {yes }}, \nu_{n o}\right)=(0,0),\left(\lambda_{\text {yes }}, \lambda_{\text {no }}\right)=(0,0)$.

Atrial fibrillation $(1)=\left(\mu_{\text {yes }}, \mu_{n o}\right)=(0,1),\left(\nu_{\text {yes }}, \nu_{n o}\right)=(0,0),\left(\lambda_{\text {yes }}, \lambda_{\text {no }}\right)=(0,0)$. 
Heart history $(1)=\left(\mu_{\text {yes }}, \mu_{n o}\right)=(0,1),\left(\nu_{\text {yes }}, \nu_{n o}\right)=(0,0),\left(\lambda_{\text {yes }}, \lambda_{\text {no }}\right)=(0,0)$.

$\operatorname{Infection}(1)=\left(\mu_{\text {yes }}, \mu_{n o}\right)=(0,1),\left(\nu_{\text {yes }}, \nu_{n o}\right)=(0,0),\left(\lambda_{\text {yes }}, \lambda_{\text {no }}\right)=(0,0)$.

Homocysteine level $(0.3)=\left(\mu_{\text {low }}, \mu_{\text {medium }}, \mu_{\text {high }}\right)=(0.7,0,0),\left(\nu_{\text {low }}, \nu_{\text {medium }}, \nu_{\text {high }}\right)=(0.1,1,1),\left(\lambda_{\text {low }}, \lambda_{\text {medium }}\right.$, $\left.\lambda_{\text {high }}\right)=(0.25,1,1)$.

Pericardial $\operatorname{cysts}(1)=\left(\mu_{y e s}, \mu_{n o}\right)=(0,1),\left(\nu_{y e s}, \nu_{n o}\right)=(0,0),\left(\lambda_{\text {yes }}, \lambda_{n o}\right)=(0,0)$.

Marfan syndrome $(1)=\left(\mu_{y e s}, \mu_{n o}\right)=(0,1),\left(\nu_{\text {yes }}, \nu_{n o}\right)=(0,0),\left(\lambda_{\text {yes }}, \lambda_{\text {no }}\right)=(0,0)$.

$\operatorname{Syphilis}(1)=\left(\mu_{\text {yes }}, \mu_{n o}\right)=(0,1),\left(\nu_{\text {yes }}, \nu_{n o}\right)=(0,0),\left(\lambda_{\text {yes }}, \lambda_{\text {no }}\right)=(0,0)$.

$\operatorname{Inflammation}(2)=\left(\mu_{\text {yes }}, \mu_{n o}\right)=(1,0),\left(\nu_{y e s}, \nu_{n o}\right)=(0,0),\left(\lambda_{\text {yes }}, \lambda_{\text {no }}\right)=(0,0)$.

$\operatorname{Clots}(2)=\left(\mu_{\text {yes }}, \mu_{n o}\right)=(1,0),\left(\nu_{\text {yes }}, \nu_{n o}\right)=(0,0),\left(\lambda_{\text {yes }}, \lambda_{n o}\right)=(0,0)$.

Cancer $(1)=\left(\mu_{\text {yes }}, \mu_{n o}\right)=(0,1),\left(\nu_{\text {yes }}, \nu_{n o}\right)=(0,0),\left(\lambda_{\text {yes }}, \lambda_{\text {no }}\right)=(0,0)$.

Electrolyte Imbalance $(1)=\left(\mu_{\text {yes }}, \mu_{\text {no }}\right)=(0,1),\left(\nu_{\text {yes }}, \nu_{\text {no }}\right)=(0,0),\left(\lambda_{\text {yes }}, \lambda_{\text {no }}\right)=(0,0)$.

\subsubsection{Explanation}

We have taken the input values from the user and with the help of mathematical equations of membership functions defined in section 3.5, we calculated the degree of truth membership functions, degree of indeterminacy membership functions, and degree of falsity membership function of each linguistic variable. The output of this module is a value lies between 0 and 1 .

\subsection{Inference Engine}

The second step is to pass the outputs of the neutrosophication module to the inference engine, and the following rules will be triggered:

R60-IF (gender=male, age=middle-age, genetic disposition=yes, smoking=yes, blood pressure $=$ high, cholesterol=high, diabetes=high, $\mathrm{BMI}=$ med, depression=high, unhealthy diet=frequently, metabolic disorder=no, physical inactivity $=$ yes, pre-eclampsia $=$ no, rheumatoid arthritis $=$ no, coffee consumption $=$ no, pregnancy $=$ no, rubella $=$ no, drugs $=$ no, tobacco $=$ yes, alcohol $=$ no, heart defect $=$ no, previous surgery $/$ injury $=$ no, thyroid $=$ no, sleep apnea $=$ no, atrial fibrillation $=$ no, heart history $=$ no, infection $=$ no, homocysteine level $=$ low, pericardial cysts $=$ no, marfan syndrome $=$ no, syphilis $=$ no, inflammation $=y e s$, clots $=y e s$, cancer $=$ no, electrolyte imbalance $=$ no) THEN (coronary artery disease=very high, cardiomyopathy=low, congenital heart disease=low, heart attack=below medium, heart arrhythmia $=$ no, peripheral artery disease=below medium, aortic disease $=$ low, pericardial disease $=$ low, deep vein thrombosis $=$ low, heart valve disease $=$ low, Heart failure $=$ low) .

R64-IF (gender =male, age=middle-age, genetic disposition=yes, smoking=yes, blood pressure=high, cholesterol=high, diabetes=high, BMI=high, depression=high, unhealthy diet=frequently, metabolic disorder=no, physical inactivity $=$ yes, pre-eclampsia $=$ no, rheumatoid arthritis $=$ no, coffee consumption $=$ no, pregnancy $=$ no, rubella $=$ no, drugs $=$ no, tobacco $=$ yes, alcohol $=$ no, heart defect $=$ no, previous surgery $/$ injury $=$ no, thyroid $=$ no, sleep apnea $=$ no, atrial fibrillation $=$ no, heart history $=$ no, infection $=$ no, homocysteine level=low, pericardial cysts $=$ no, marfan syndrome $=$ no, syphilis $=$ no, inflammation $=$ yes, clots=yes, cancer $=$ no, Electrolyte Imbalance=no) THEN (coronary artery disease=very high, cardiomyopathy=low, congenital heart disease=low, 
heart attack=below medium, heart arrhythmia $=$ no, peripheral artery disease=below medium, aortic disease $=$ low, pericardial disease $=$ low, deep vein thrombosis $=$ low, heart valve disease $=$ low, heart failure $=$ low $)$.

Table 2 shows the result of the inference engine.

Table 2: Results of the Inference Engine

\begin{tabular}{|c|c|c|}
\hline Input & $\overline{\mathbf{R 6 0}}(\mu, \nu, \lambda)$ & $\overline{\mathbf{R 6 4}}(\mu, \nu, \lambda)$ \\
\hline Gender & male $(1,0,0)$ & male $(1,0,0)$ \\
\hline Age & middle $(0.67,0.17,0.4)$ & middle $(0.67,0.17,0.4)$ \\
\hline Genetic disposition & $\operatorname{yes}(1,0,0)$ & yes $(1,0,0)$ \\
\hline Smoking & yes $(1,0,0)$ & yes $(1,0,0)$ \\
\hline Blood pressure & high $(0.8,0.4,0.29)$ & high $(0.8,0.4,0.29)$ \\
\hline Cholesterol & high $(0.21,0.81,0.8)$ & high $(0.21,0.81,0.8)$ \\
\hline Diabetes & high $(0.5,0.58,0.53)$ & high $(0.5,0.58,0.53)$ \\
\hline BMI & med $(0,0.75,1)$ & high $(0.86,0.57,0.2)$ \\
\hline Depression & high $(0.8,0.33,0.25)$ & high $(0.8,0.33,0.25)$ \\
\hline Unhealthy diet & frequently $(0.5,0.83,0.625)$ & frequently $(0.5,0.83,0.625)$ \\
\hline Metabolic disorder & no $(1,0,0)$, & no $(1,0,0)$ \\
\hline Physical inactivity & yes $(1,0,0)$ & yes $(1,0,0)$ \\
\hline Pre-eclampsia & no $(1,0,0)$ & no $(1,0,0)$ \\
\hline Rheumatoid arthritis & no $(1,0,0)$ & no $(1,0,0)$ \\
\hline Coffee consumption & no $(1,0,0)$ & no $(1,0,0)$ \\
\hline Pregnancy & no $(1,0,0)$ & no $(1,0,0)$ \\
\hline Rubella & no $(1,0,0)$ & no $(1,0,0)$ \\
\hline Drugs & no $(1,0,0)$ & no $(1,0,0)$ \\
\hline Tobacco & yes $(1,0,0)$ & yes $(1,0,0)$ \\
\hline Alcohol & no $(1,0,0)$ & no $(1,0,0)$ \\
\hline heart defect & no $(1,0,0)$ & no $(1,0,0)$ \\
\hline Previous surgery/injury & no $(1,0,0)$ & no $(1,0,0)$ \\
\hline Thyroid & no $(1,0,0)$ & no $(1,0,0)$ \\
\hline Sleep apnea & no $(1,0,0)$ & no $(1,0,0)$ \\
\hline Atrial fibrillation & no $(1,0,0)$ & no $(1,0,0)$ \\
\hline Heart history & no $(1,0,0)$ & no $(1,0,0)$ \\
\hline Infection & no $(1,0,0)$ & no $(1,0,0)$ \\
\hline Homocysteine level & low $(0.7,0.1,0.25)$ & low $(0.7,0.1,0.25)$ \\
\hline Pericardial cysts & no $(1,0,0)$ & no $(1,0,0)$ \\
\hline Marfan syndrome & no $(1,0,0)$ & no $(1,0,0)$ \\
\hline Syphilis & no $(1,0,0)$ & no $(1,0,0)$ \\
\hline Inflammation & yes $(1,0,0)$ & yes $(1,0,0)$ \\
\hline Clots & yes $(1,0,0)$ & yes $(1,0,0)$ \\
\hline Cancer & no $\left(\begin{array}{lll}1 & 0 & 0\end{array}\right)$ & no $(1,0,0)$ \\
\hline Electrolyte Imbalance & no $(1,0,0)$ & no $(1,0,0)$ \\
\hline$(\min , \max , \max )$ & $(0,0.83,1)$ & $(0.21,0.83,0.8)$ \\
\hline
\end{tabular}

We can ignore R60 because the inference engine result shows that its truth membership is absolutely zero and falsity membership is 1 . 


\subsubsection{Explanation}

The value of each membership function is passed to the inference engine and find out the active rules. According to the specified example, rule 60 and 64 triggers and their values represent the firing strength of both rules as shown in Table 2.

\subsection{De-neutrosophication}

The last step of the proposed system is de-neutrosophication to get the final output. Now we will perform de-neutrosophication using two methods:

a). By using the de-neutrosophication method proposed in [9].

low $=(0,0,1 ; 0.3,1.2,1.2 ; 0.2,0.9,0.9)=0+2(0)+1+0.3+2(1.2)+1.2+0.2+2(0.9)+0.9 / 12=0.605$.

below medium $=(0,1,2 ; 0.2,1.5,2.5 ; 0.1,1,1.7)=0+2(1)+2+0.2+2(1.5)+2.5+0.1+2(1)+1.7 / 12=1.125$.

very high $=(4,5,5 ; 4.3,4.3,5,4.2,4.2,5)=4+2(5)+5+4.3+2(4.3)+5+4.2+2(4.2)+5 / 12=4.54$.

We concluded that the maximum risk value is of coronary artery disease.

b). According to our de-neutrosophication method the final output is calculated as follows:

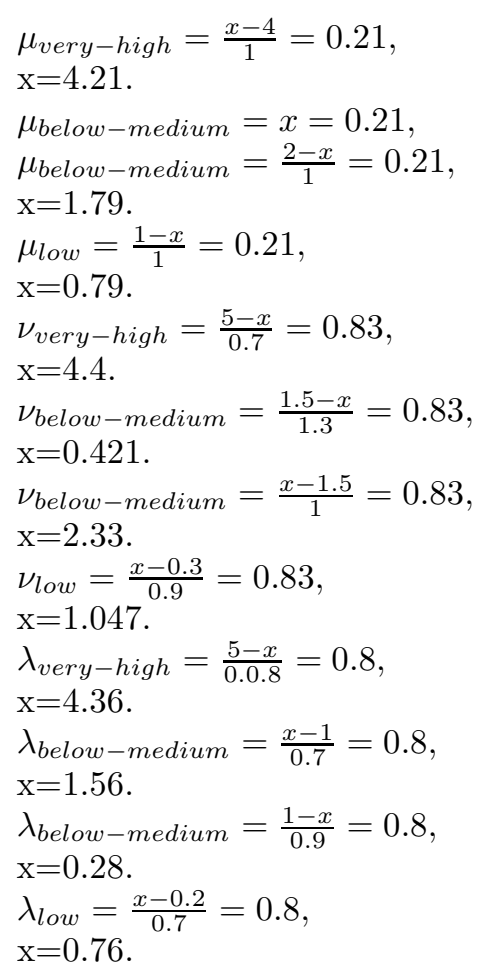

Here,

$\mathrm{P}=$ Point in the sample space,

$\mathrm{TR}=$ Degree of truth at $\mathrm{P}$,

$\mathrm{IN}=$ Degree of indeterminacy at $\mathrm{P}$,

$\mathrm{FL}=$ Degree of falsity at $\mathrm{P}$,

$\mathrm{U}=3-(\mathrm{TR}+\mathrm{IN}+\mathrm{FL})$, 


\section{$\mathrm{V}=\mathrm{TR}^{*} \mathrm{IN}^{*} \mathrm{FL}$}

Table 3, Table 4, and Table 5 show the de-neutrosophication results of rule 65 .

Table 3: De-neutrosophication of very high

\begin{tabular}{|c|c|c|c|c|c|c|c|}
\hline $\mathbf{P}$ & TR & IN & FL & $\mathbf{U}$ & $\mathbf{V}$ & $\mathbf{U}+\mathbf{V}$ & $\mathbf{P}(\mathbf{U}+\mathbf{V})$ \\
\hline 4 & 0 & 1 & 1 & 1 & 0 & 1 & 4 \\
\hline 4.1 & 0.1 & 1 & 1 & 0.9 & 0.1 & 1 & 4.1 \\
\hline 4.2 & 0.2 & 1 & 0.8 & 1 & 0.16 & 1.16 & 4.872 \\
\hline 4.3 & 0.21 & 0.83 & 0.8 & 1.16 & 0.14 & 1.3 & 5.6 \\
\hline 4.4 & 0.21 & 0.83 & 0.75 & 1.21 & 0.13 & 1.34 & 5.9 \\
\hline 4.5 & 0.21 & 0.714285714 & 0.625 & 1.450714286 & 0.09375 & 1.544464286 & 6.950089286 \\
\hline 4.6 & 0.21 & 0.571428571 & 0.5 & 1.718571429 & 0.06 & 1.778571429 & 8.181428571 \\
\hline 4.7 & 0.21 & 0.428571429 & 0.375 & 1.986428571 & 0.03375 & 2.020178571 & 9.494839286 \\
\hline 4.8 & 0.21 & 0.285714286 & 0.25 & 2.254285714 & 0.015 & 2.269285714 & 10.89257143 \\
\hline 4.9 & 0.21 & 0.142857143 & 0.125 & 2.522142857 & 0.00375 & 2.525892857 & 12.376875 \\
\hline 5 & 0.21 & 0 & 0 & 2.79 & 0 & 2.79 & 13.95 \\
\hline & & & & & sum & 18.73 & 86.312 \\
\hline & & & & & & Final & 4.61 \\
\hline
\end{tabular}

Table 4: De-neutrosophication of below medium

\begin{tabular}{|c|c|c|c|c|c|c|c|}
\hline $\mathbf{P}$ & TR & IN & FL & $\mathbf{U}$ & $\mathbf{V}$ & $\mathbf{U}+\mathbf{V}$ & $\mathbf{P}(\mathbf{U}+\mathbf{V})$ \\
\hline 0 & 0 & 1 & 1 & 1 & 0 & 1 & 0 \\
\hline 0.2 & 0.2 & 0.83 & 0.8 & 1.17 & 0.1328 & 1.3028 & 0.26056 \\
\hline 0.4 & 0.4 & 0.83 & 0.666666667 & 1.1 & 0.22 & 1.32 & 0.529 \\
\hline 0.6 & 0.6 & 0.692307692 & 0.444444444 & 1.263247863 & 0.184615385 & 1.447863248 & 0.868717949 \\
\hline 0.8 & 0.8 & 0.538461538 & 0.222222222 & 1.439316239 & 0.095726496 & 1.535042735 & 1.228034188 \\
\hline 1 & 1 & 0.384615385 & 0 & 1.615384615 & 0 & 1.615384615 & 1.615384615 \\
\hline 1.2 & 0.8 & 0.230769231 & 0.285714286 & 1.683516484 & 0.052747253 & 1.736263736 & 2.083516484 \\
\hline 1.4 & 0.6 & 0.076923077 & 0.571428571 & 1.751648352 & 0.026373626 & 1.778021978 & 2.489230769 \\
\hline 1.6 & 0.4 & 0.1 & 0.8 & 1.7 & 0.032 & 1.732 & 2.7712 \\
\hline 1.8 & 0.2 & 0.3 & 0.8 & 1.7 & 0.048 & 1.748 & 3.1464 \\
\hline 2 & 0 & 0.5 & 0.8 & 1.7 & 0 & 1.7 & 3.4 \\
\hline 2.2 & 0 & 0.7 & 0.8 & 1.5 & 0 & 1.5 & 3.3 \\
\hline 2.4 & 0 & 0.83 & 0.8 & 1.3 & 0 & 1.3 & 3.12 \\
\hline 2.5 & 0 & 0.83 & 0.8 & 1.2 & 0 & 1.2 & 3 \\
\hline & & & & & sum & 20.93 & 27.82 \\
\hline & & & & & & Final & 1.33 \\
\hline
\end{tabular}


Table 5: De-neutrosophication of low

\begin{tabular}{|c|c|c|c|c|c|c|c|}
\hline $\mathbf{P}$ & TR & $\mathbf{I N}$ & $\mathbf{F L}$ & $\mathbf{U}$ & $\mathbf{V}$ & $\mathbf{U}+\mathbf{V}$ & $\mathbf{P}(\mathbf{U}+\mathbf{V})$ \\
\hline 0 & 0.21 & 1 & 1 & 0.79 & 0.21 & 1 & 0 \\
\hline 0.1 & 0.21 & 1 & 1 & 0.79 & 0.21 & 1 & 0.1 \\
\hline 0.2 & 0.21 & 1 & 0 & 1.79 & 0 & 1.79 & 0.358 \\
\hline 0.3 & 0.21 & 0 & 0.142857143 & 2.647142857 & 0 & 2.647142857 & 0.794142857 \\
\hline 0.4 & 0.21 & 0.111111111 & 0.285714286 & 2.393174603 & 0.006666667 & 2.39984127 & 0.959936508 \\
\hline 0.5 & 0.21 & 0.222222222 & 0.428571429 & 2.139206349 & 0.02 & 2.159206349 & 1.079603175 \\
\hline 0.6 & 0.21 & 0.333333333 & 0.571428571 & 1.885238095 & 0.04 & 1.925238095 & 1.155142857 \\
\hline 0.7 & 0.21 & 0.444444444 & 0.714285714 & 1.631269841 & 0.066666667 & 1.697936508 & 1.188555556 \\
\hline 0.8 & 0.2 & 0.555555556 & 0.8 & 1.444444444 & 0.088888888 & 1.5333333333 & 1.226666667 \\
\hline 0.9 & 0.1 & 0.666666667 & 0.8 & 1.433333333 & 0.053333333 & 1.486666667 & 1.338 \\
\hline 1 & 0 & 0.7777777778 & 0.8 & 1.4222222222 & 0 & 1.4222222222 & 1.422222222 \\
\hline 1.1 & 0 & 0.83 & 0.8 & 1.37 & 0.664 & 2.034 & 2.2374 \\
\hline 1.2 & 0 & 0.83 & 0.8 & 1.37 & 0.664 & 2.034 & 2.4408 \\
\hline & & & & & sum & 23.138 & 14.3062 \\
\hline & & & & & & Final & 0.62 \\
\hline
\end{tabular}

Take the maximum value out of all outputs, and the risk of coronary artery disease is again very high. Hence, we concluded that both methods give the same output.

\subsubsection{Explanation}

Now, de-neutrosophication has been performed. We have used two ways to do so. The first method is taken from the literature and the second method is proposed by us. Using both methods, we calculated the risk of eleven CVDs which shown the risk of each disease.

\subsection{Three-layered causal hierarchy}

According to Pearl et al., there are three Layers of causal hierarchy to measure the quality of explanation [34]-[35]:

Level 1: Association- How much mentioned risk factors belongs to the specified cardiovascular disease? This question is asked by medical experts and concluded that all the mentioned risk factors are closely related to CVDs.

Level 2: Intervention- What if the medical professional used the proposed system- will the patient get diagnosed earlier? According to the experts, the proposed system helps medical professionals a lot to diagnose the risk of CVDs at the earliest.

Level 3: Counterfactuals- Was it the cardiovascular disease that causes the specified risk factor? After comparing the results of risk with risk factors, it is concluded that CVDs causes most of the risk factors. The CVDs risk is closely linked with defined risk factors.

\section{Comparison Analysis}

This section provides a comparative analysis of the proposed de-neutrosophication formula with the existing de-neutrosophication formula, fuzzy soft-sets, and fuzzy cognitive maps with the help of various data sets [9], [37]-[38]. In the literature, many methods of decision making are discussed. Here we considered fuzzy soft sets and fuzzy cognitive maps as decision-making approaches to compare the proposed de-neutrosophication method. We've taken twenty different data sets to test the accuracy of our system. The results obtained by these methods are very similar to the results produced by our method. All methods identified the same heart 
disease against the same dataset values. The final risk values obtained by these methods are summarized in Figure 12.

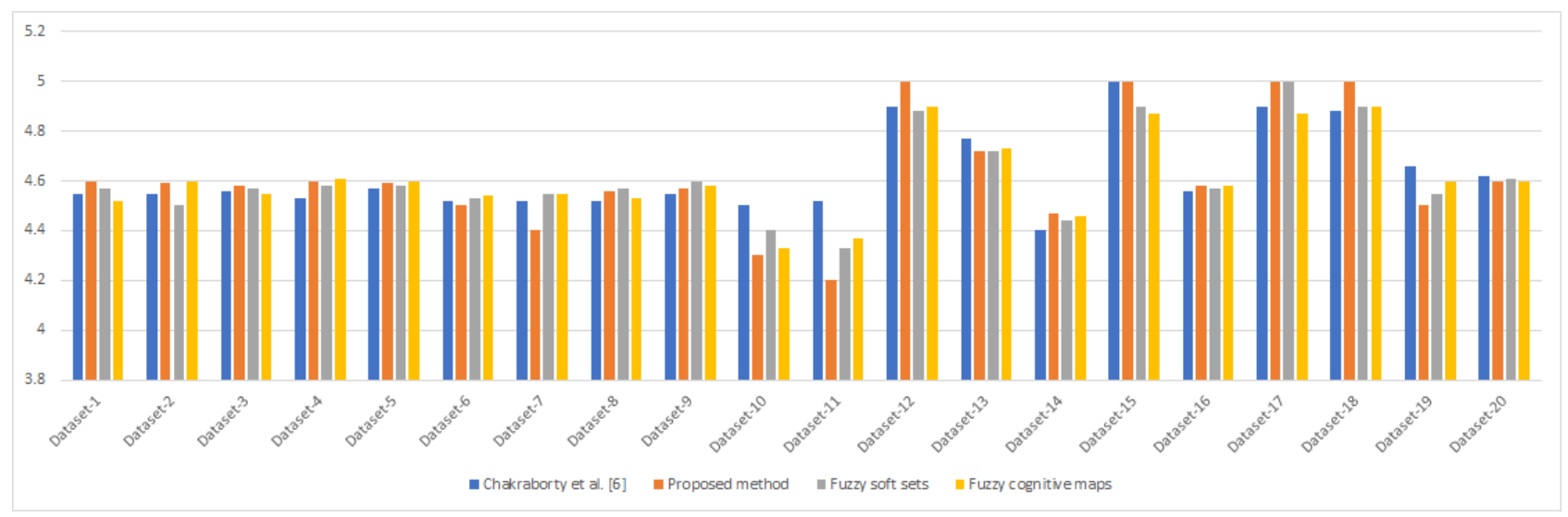

Figure 12: Comparison analysis

The proposed system discusses the practical approach of applying neutrosophic logic to cardiovascular diseases. Based on the concept of neutrosophic sets, its advanced decisive alternatives that would allow us (and the machines) to consider the complex role of biological phenomena, thereby meeting real medical and experimental needs. This system aims to facilitate doctors and emergency specialists. Particularly in times of crisis, this system can also be extended to increase alarm for any deviation monitored.

\section{Conclusion and future directions}

Multiple attribute decision-making method is an algorithm that is commonly used and needs certain aggregation methods. In this paper, we have purposed the neutrosophic clinical decision-making system for cardiovascular diseases using explainable AI approaches. Our system helps medical experts to early detect the risk of CVDs so that precautionary measures can be taken timely. As a result, overall mortality will be declined. Our system takes thirty-five parameters as inputs and determines the risks of eleven cardiovascular diseases. There are five main modules of the system which are neutrosophication, knowledge base, inference engine, de-neutrosophication, and explainability. We have used an ante-hoc explainable artificial intelligence method to make this system more understandable and also used a three-layered causability hierarchy to measures the quality of explanation. We have designed an algorithm for a better understanding of the decision-making process, and computed its time complexity. We have proposed a new de-neutrosophication method. To check the accuracy of this method, we consider an example and perform the de-neutrosophication process using the traditional method and the proposed de-neutrosophication method. Also, we demonstrated a comparative study with other decision-making methods. There are many multi-criteria decision-making methods discussed in the literature. These methods can also be applied to cardiovascular disease risk analysis. The proposed approach can be further applied in many other problems like early diagnosis of cancer, types of fevers, and other diseases as well. Our proposed work is applicable in all those domains that undergo the decision-making process, for example, in precision agriculture, manufacturing industries, robotics, gaming, the textile industry, and many more.

Conflict of Interest: The authors declare that they have no conflict of interest. 


\section{References}

[1] Shumaiza, Akram, M. and Ahmad N. Al-Kenani, Multiple-attribute decision making ELECTRE II method under bipolar fuzzy model, Algorithms, 12(11)(2019), 226.

[2] Akram, M., Ishfaq, N., Sayed, S., and Smarandache, F., Decision-making approach based on neutrosophic rough information, Algorithms, 11(5)(2018), 59.

[3] Habib, S., and Akram, M., and Ashraf, A., Fuzzy climate decision support systems for tomatoes in high tunnels, International Journal of Fuzzy Systems, 19(3)(2017), 751-775.

[4] Gade, K., Geyik, S. C., Kenthapadi, K., Mithal, V., and Taly, A., Explainable AI in industry, International Conference on Knowledge Discovery \& Data Mining, ACM, (2019), 3203-3204.

[5] Holzinger, A., Langs, G., Denk, H., Zatloukal, K., and Müller, H., Causability and explainability of artificial intelligence in medicine, Wiley Interdisciplinary Reviews: Data Mining and Knowledge Discovery, 9(4)(2019), p. e1312.

[6] Holzinger, A., Carrington, A., and Müller, H., Measuring the quality of explanations: the system causability scale (SCS), KI-Künstliche Intelligenz, (2020), 1-6.

[7] Lamy, J. B., Sekar, B., Guezennec, G., Bouaud, J., and Séroussi, B., Explainable artificial intelligence for breast cancer: A visual case-based reasoning approach, Artificial Intelligence in Medicine, 94(2019), 42-53.

[8] Zadeh, L.A., Fuzzy sets, Information and Control, 8(3)(1965), 338-353.

[9] Chakraborty, A., Mondal, S.P., Ahmadian, A., Senu, N., Alam, S., and Salahshour, S. Different forms of triangular neutrosophic numbers, de-neutrosophication techniques, and their applications, Symmetry, 10(8)(2018), 327.

[10] Smarandache, F., A unifying field in logics. neutrosophy: Neutrosophic probability, set and logic, (1999), $1-141$.

[11] Akram, M., Single-valued neutrosophic graphs, Infosys Science Foundation Series in Mathematical Sciences, Springer, (2018).

[12] Akram, M., Naz, S., Smarandache, F., Generalization of maximizing deviation and TOPSIS method for MADM in simplified neutrosophic hesitant fuzzy environment, Symmetry, 11(8)(2019), 1058.

[13] Wang, H., Smarandache, F., Zhang, Y., and Sunderraman, R., Single-valued neutrosophic sets, Multispace Multistruct, 4(2010), 410-413.

[14] Ye, J., A multicriteria decision-making method using aggregation operators for simplified neutrosophic sets, Journal of Intelligent and Fuzzy Systems, 26(5)(2014), 2459-2466.

[15] Ashraf, S., Abdullah, S., Zeng, S., Jin, H., and Ghani, F. Fuzzy decision support modeling for hydrogen power plant selection based on single valued neutrosophic sine trigonometric aggregation operators, Symmetry, 12(2020), 298.

[16] Garg, H., and Nancy, Multiple attribute decision making based on immediate probabilities aggregation operators for single-valued and interval neutrosophic sets, Journal of Applied Mathematics and Computing, 63(2020), 619-653.

[17] Garg, H., and Nancy, Algorithms for single-valued neutrosophic decision making based on TOPSIS and clustering methods with new distance measure, AIMS Mathematics, 5(3)(2020), 2671-2693.

[18] Garg, H., and Nancy, Multiple criteria decision making based on Frank Choquet Heronian mean operator for single-valued neutrosophic sets, Applied and Computational Mathematics, 18(2)(2019), 163-188. 
[19] Garg, H., and Nancy, New logarithmic operational laws and their applications to multiattribute decision making for single-valued neutrosophic numbers, Cognitive Systems Research, 52(2018), 931-946.

[20] Zhu, X., Bai, K., Wang, J., Zhang, R., and Xing, Y., Pythagorean fuzzy interaction power partitioned Bonferroni means with applications to multi-attribute group decision making, Journal of Intelligent \& Fuzzy Systems, 36(4)(2019), 3423-3438.

[21] Peng, J.J., Wang, J.Q., Wang, J., Zhang, H.Y., and Chen, X.H., Simplified neutrosophic sets and their applications in multi-criteria group decision making problems, International Journal of Systems Science, $\mathbf{4 7}(10)(2016), 2342-2358$.

[22] Peng, J.J., Wang, J.Q., Wu, X.H., Zhang, H.Y., and Chen, X.H., The fuzzy cross-entropy for intuitionistic hesitant fuzzy sets and their applicationin multi-criteria decision-making, International Journal of Systems Science, 46(13)(2015), 2335-2350.

[23] Ye, J., and Fu, J., Multi-period medical diagnosis method using a single-valued neutrosophic similarity measure based on tangent function, Computer Methods and Programs in Biomedicine, 123(2016), 142-149.

[24] Cui, W.H., and Jun, Y., Logarithmic similarity measure of dynamic neutrosophic cubic sets and its application in medical diagnosis, Computers in Industry, 111(2019), 198-206.

[25] Abdel-Basset, M., Manogaran, G., Gamal, A., and Smarandache, F., A group decision making framework based on neutrosophic TOPSIS approach for smart medical device selection, Journal of Medical Systems, 43(2)(2019), 38 .

[26] Guleria, A., Srivastava, S., and Bajaj, R. K., On parametric divergence measure of neutrosophic sets with its application in decision-making models, Neutrosophic Sets and Systems, 29(2019), 101-120.

[27] Smarandache, F., A unifying field in Logics: neutrosophic logic, Philosophy. American Research Press, (1999), 1-141.

[28] https://www.mayoclinic.org/diseases-conditions/heart-disease/symptoms-causes/syc-20353118.

[29] https://ada.com/cardiovascular-disease-risk-factors/.

[30] https://www.who.int/news-room/fact-sheets/detail/cardiovascular-diseases-(cvds).

[31] https://www.world-heart-federation.org/resources/risk-factors/.

[32] Aggarwal, S., Biswas, R., and Ansari, A. Q., Neutrosophic modeling and control, International Conference on Computer and Communication Technology (ICCCT), IEEE, (2010), 718-723.

[33] Angelov, P., Crispification: defuzzification over intuitionistic fuzzy sets, Bulletin for Studies and Exchanges on Fuzziness and its Applications, BUSEFAL, 64 (1995), 51-55.

[34] Pearl, J., Theoretical impediments to machine learning with seven sparks from the causal revolution, (2018), arXiv preprint arXiv:1801.04016.

[35] Pearl, J., and Mackenzie, D., The book of why: the new science of cause and effect, Basic Books, (2018).

[36] Butt, M. A. and Akram, M., A novel fuzzy decision making system for cpu scheduling algorithm, Neural Computing \& Applications, 27(7)(2016), 1927-1939.

[37] Habib, S., and Akram, M., Diagnostic methods and risk analysis based on fuzzy soft information, International Journal of Biomathematics, 11(8)(2018), 1850096.

[38] Habib, S., and Akram, M., Medical decision support systems based on fuzzy cognitive maps, International Journal of Biomathematics, 12(6)(2019), 1950069. 